\title{
Pseudo-differential operators on fractals and other metric measure spaces
}

\author{
Marius Ionescu, Luke G. Rogers and Robert S. Strichartz
}

\begin{abstract}
We define and study pseudo-differential operators on a class of fractals that include post-critically finite (p.c.f.) self-similar sets and Sierpiński carpets. Using sub-Gaussian estimates for the heat operator we prove that our operators have kernels that decay and, in the constant coefficient case, are smooth off the diagonal. Our analysis can be extended to products of fractals. While our results are applicable to a larger class of metric measure spaces with Laplacian, we use them to study elliptic, hypoelliptic, and quasi-elliptic operators on p.c.f. fractals, answering a few open questions posed in a series of recent papers. We extend our class of operators to include the so called Hörmander hypoelliptic operators and we initiate the study of wavefront sets and microlocal analysis on p.c.f. fractals.
\end{abstract}

\section{Introduction}

In this paper we define and study pseudo-differential operators on metric measure spaces endowed with a nonpositive self-adjoint Laplacian such that the heat operator satisfies sub-Gaussian estimates. The motivating examples for our work are metric measure spaces constructed from p.c.f. fractals ([21], [37], [34]). However, our results capture known facts from classical harmonic analysis on $\mathbb{R}^{n}$ and Riemannian manifolds and can be used to define pseudo-differential operators on other metric measure spaces. For example, the hypotheses of our main theorems apply to the operators defined in [16].

Analysis on fractals from either the probabilistic or the analytic viewpoint has been the focus of intense study recently (see, for example, [15], [4], [21], [39] and references therein). Several recent papers have studied properties of spectral operators on fractals. For example, [38] shows some new convergence properties of

Mathematics Subject Classification (2010): Primary 35S05, 35P99, 46F12; Secondary 28C15, $58 \mathrm{C} 40$.

Keywords: Pseudo-differential operators, fractals, self-similar, elliptic, hypoelliptic, quasi-elliptic, Laplacian, metric measure space, sub-Gaussian heat kernel estimates, wavefront set, microlocal analysis. 
Fourier series on fractals with spectral gaps and establishes a Littlewood-Paley inequality for such fractals. In [20] a kernel formula for the resolvent of the Laplacian on any p.c.f. fractal is given; this generalizes Kigami's result for the Green function on p.c.f. fractals ([21]). These results have been extended to infinite blowups of fractals in [24]. Numerical results suggest that this result might hold for other spectral operators on fractals [1], [7]. The main result of [19] says that if a spectral operator on a p.c.f. fractal is given by integration with respect to a kernel that is smooth and has a specific type of decay off the diagonal, then it is a Calderón-Zygmund operator in the sense of [31] (Section I.6.5). In particular, the authors show that the Riesz and Bessel potentials and, more generally, the Laplace type operators are Calderón-Zygmund operators. On the other hand, in [11], [28], the authors show that spectral multiplier operators on metric measure spaces and products of such spaces are bounded on $L^{q}$ as long as the heat operator satisfies specific estimates. The Laplacian defined on some p.c.f. fractals and some highly symmetric Sierpiński gaskets satisfies these assumptions.

We begin by presenting the standing hypotheses for our results in Section 2. We also briefly review the definition and main properties of p.c.f. fractals and the Laplacians defined on them.

The pseudo-differential operators with constant coefficients that are studied in Section 3 generalize the class of spectral multipliers studied in the papers mentioned above. We justify symbolic calculus for these operators and show that they are given by integration with respect to kernels that are smooth and decay off the diagonal, extending some of the results of [19]. Moreover, pseudo-differential operators of order 0 on p.c.f. fractals are Calderón-Zygmund operators, and thus extend to bounded operators on $L^{q}$, for all $1<q<\infty$. In this context we therefore recover the results of [11] and [28]. We extend our analysis to products of such spaces in Section 5.

We define Sobolev spaces on spaces built out of p.c.f. fractals in Section 4 and prove that pseudo-differential operators with constant coefficients are bounded on them. We study elliptic and hypoelliptic operators on fractals in Section 6. Namely, we prove that a pseudo-differential operator satisfies the pseudo-local properties and that an elliptic operator is hypoelliptic. This gives positive answers to some open questions posed in [39], [6], [25]. An interesting class of operators that can be defined on fractals with spectral gaps are the so called quasi-elliptic operators $([6],[28])$. We show that every quasi-elliptic operator is equal to an elliptic pseudodifferential operator, though there are quasi-elliptic differential operators which are not elliptic as differential operators.

In Section 7 we extend the class of pseudo-differential operators to include operators for which the derivatives of the symbols have a slower rate of decay. As an application we show that the Hörmander type hypoelliptic operators are hypoelliptic. This extends one side of the classical result of [41] (Theorem 2.1 in Chapter III). The converse is false in general, as is exemplified by the quasi-elliptic operators (see Subsection 6.1).

Section 8 introduces the wavefront set and microlocal analysis on products of compact spaces built out of fractals. We show that pseudo-differential operators 
may decrease the wavefront set and that elliptic operators preserve the wavefront set, extending results from classical harmonic analysis (see, for example, [29]). We also describe the wavefront set for a few specific examples.

In the last section, we study properties of pseudo-differential operators with variable coefficients on compact fractafolds. The main results of the section are the continuity off the diagonal of the kernels of such operators and the $L^{q}$-boundedness of these operators. These results cannot be obtained using the methods of [11] and [28]. We also conjecture that the pseudo-differential operators with variable coefficients of order 0 are Calderón-Zygmund operators.

Some of the results proved here are extensions of the corresponding results from classical harmonic analysis (see, for example [41], [30], [31]). However the proofs of our results are very different. The main reason for this difference is that the product of smooth functions is, in general, no longer in the domain of the Laplacian [5]. Therefore techniques that are essential in real analysis like multiplication with a smooth bump are not available to us. We frequently use the Borel type theorem proved in [26] to decompose a smooth function in a sum of smooth functions.

Acknowledgements. The authors would like to thank Camil Muscalu and Alexander Teplyaev for many useful conversations during the preparation of the manuscript. They also thank the referee for various suggestions, including pointing out the relevance of [16] to this topic. The authors express their thanks to Yin-Tat Lee for pointing out an error in an earlier draft of this article.

\section{Background}

For the main results of Sections 3 and 5 we need a metric space $(X, R)$ with a Borel measure $\mu$ and a negative self-adjoint Laplacian $\Delta$. We assume that $X$ satisfies the doubling condition, that is, there is a constant $C>0$ such that

$$
\mu(B(x, 2 r)) \leq C \mu(B(x, r)) \text { for all } x \in X \text { and } r>0 .
$$

We also assume that the heat operator $e^{t \Delta}$ has a positive kernel $h_{t}(x, y)$ that satisfies the sub-Gaussian upper estimate

$$
h_{t}(x, y) \leq c_{1} t^{-\beta} \exp \left(-c_{2}\left(\frac{R(x, y)^{d+1}}{t}\right)^{\gamma}\right)
$$

where $c_{1}, c_{2}>0$ are constants independent of $t, x$ and $y$. In this expression both $d$ and $\gamma$ are constants that depend on $X$ and $\beta=d /(d+1)$. Moreover we assume that $h_{z}(x, y)$ is a holomorphic function on $\{\operatorname{Re} z>0\}$.

Examples of spaces that satisfy the above hypothesis include the so called p.c.f. fractals and the highly symmetric Sierpiński carpets. We review some of the definitions and properties of the p.c.f. fractals and of the Laplacians defined on them. For more details the reader can consult the books [21] and [39]. Recall that an iterated function system (i.f.s.) is a collection $\left\{F_{1}, \ldots, F_{N}\right\}$ of contractions on $\mathbb{R}^{d}$. For such an i.f.s. there exists a unique self-similar set $K$ satisfying (see [18])

$$
K=F_{1}(K) \cup \cdots \cup F_{N}(K) .
$$


For $\omega_{1}, \omega_{2}, \ldots \omega_{n} \in\{1, \ldots, N\}, \omega=\omega_{1} \omega_{2} \ldots \omega_{n}$ is a word of length $n$ over the alphabet $\{1, \ldots, N\}$. The subset $K_{\omega}=F_{\omega}(K):=F_{\omega_{1}} \circ \cdots \circ F_{\omega_{n}}(K)$ is called a cell of level $n$. The set of all finite words over $\{1, \ldots, N\}$ is denoted by $W_{*}$. Each map $F_{i}$ of the i.f.s. defining $K$ has a unique fixed point $x_{i}$. We say that $K$ is a post-critically finite (p.c.f.) self-similar set if there is a subset $V_{0} \subseteq\left\{x_{1}, \ldots, x_{N}\right\}$ satisfying

$$
F_{\omega}(K) \cap F_{\omega^{\prime}}(K) \subseteq F_{\omega}\left(V_{0}\right) \cap F_{\omega^{\prime}}\left(V_{0}\right)
$$

for any $\omega \neq \omega^{\prime}$ having the same length. The set $V_{0}$ is called the boundary of $K$ and the boundary of a cell $K_{\omega}$ is $F_{\omega}\left(V_{0}\right)$. One defines $V_{1}=\bigcup_{i} F_{i}\left(V_{0}\right)$, and, inductively, $V_{n}=\bigcup_{i} F_{i}\left(V_{n-1}\right)$ for $n \geq 2$. The fractal $K$ is the closure of $\bigcup_{n} V_{n}$.

The Laplacian on p.c.f. fractals may be built using Kigami's construction [21] from a self-similar Dirichlet energy form $\mathcal{E}$ on $K$ with weights $\left\{r_{1}, \ldots, r_{N}\right\}$ :

$$
\mathcal{E}(u)=\sum_{i=1}^{N} r_{i}^{-1} \mathcal{E}\left(u \circ F_{i}\right) .
$$

The existence of such forms is non-trivial, but on a large collection of examples they may be obtained from the approximating graphs as the appropriate renormalized limit of graph energies ([21], [39]).

The second ingredient is the existence of a unique self-similar measure

$$
\mu(A)=\sum_{i=1}^{N} \mu_{i} \mu\left(F^{-1}(A)\right),
$$

where $\left\{\mu_{1}, \ldots, \mu_{N}\right\}$ are weights such that $0<\mu_{i}<1$ and $\sum \mu_{i}=1$, see [18].

Then the Laplacian is defined weakly: $u \in \operatorname{dom} \Delta_{\mu}$ with $\Delta_{\mu} u=f$ if

$$
\mathcal{E}(u, v)=-\int_{X} f v d \mu
$$

for all $v \in \operatorname{dom} \mathcal{E}$ with $\left.v\right|_{V_{0}}=0$. The domain of the Laplacian depends on the assumptions that one makes about $f$. Kigami ([21]) assumes that $f$ is continuous, but in this paper it will be more natural to assume that $f$ is in $L^{2}(\mu)$, which gives a Sobolev space (see Section 4). We write $u \in \operatorname{dom}_{L^{p}} \Delta$ if $f \in L^{p}(\mu)$.

The effective resistance metric $R(x, y)$ on $K$ is defined via

$$
R(x, y)^{-1}=\min \{\mathcal{E}(u): u(x)=0 \text { and } u(x)=1\} .
$$

It is known that the resistance metric is topologically equivalent, but not metrically equivalent to the Euclidean metric ([21], [39]).

The unit interval and the Sierpiński gasket ([21], [39], [13], [5], [23], [35], [42]) are important examples of p.c.f. fractals. Other examples are the affine nested fractals [12] that in turn are generalizations of the nested fractals [22].

Some of the spaces that we consider in this paper are built from p.c.f. fractals as in [34], [35]. In those papers the author defines fractal blow-ups of a p.c.f. fractal $K$ and fractafolds based on $K$. The former generalizes the relationship between the 
unit interval and the real line to arbitrary p.c.f. self-similar sets, while the latter is the natural analogue of a manifold. Let $w \in\{1, \ldots, N\}^{\infty}$ be an infinite word. Then

$$
F_{w_{1}}^{-1} \ldots F_{w_{m}}^{-1} K \subseteq F_{w_{1}}^{-1} \ldots F_{w_{m}}^{-1} F_{w_{m}+1}^{-1} K
$$

The fractal blow-up is

$$
X=\bigcup_{m=1}^{\infty} F_{w_{1}}^{-1} \ldots F_{w_{m}}^{-1} K .
$$

If $C$ is an $n$ cell in $K$, then $F_{w_{1}}^{-1} \ldots F_{w_{m}}^{-1} C$ is called an $(n-m)$ cell. The blow-up depends on the choice of the infinite word $w$. In general there are an uncountably infinite number of blow-ups which are not homeomorphic. In this paper we assume that the infinite blow-up $X$ has no boundary. This happens unless all but a finite number of letters in $w$ are the same. One can extend the definition of the energy $\mathcal{E}$ and measure $\mu$ to $X$. The measure $\mu$ will be $\sigma$-finite rather than finite. Then one can define a Laplacian on $X$ by the weak formulation. It is known that, for a large class of p.c.f. fractals, the Laplacian on an infinite blow-up without boundary has pure point spectrum $([42],[27])$.

One can view a fractal blow-up as a collection of copies of $K$ that are glued together at boundary points. Every point of $X$ has a neighborhood which is homeomorphic to a neighborhood of a point in $K$. A fractafold [35] based on $K$ is defined to be a set which satisfies this latter property. We will work on a restricted class of fractafolds, which may be thought of as more like triangulated manifolds. Specifically we will consider fractafolds $X$ that consist of a finite or infinite union of copies of $K$ glued together at some of the boundary points. The fractafold $X$ is compact if and only if we consider a finite number of copies of $K$. We suppose in the following that all the copies of $K$ have the same size in $X$. If all the boundary points of the copies of $K$ are paired, then the fractafold $X$ has no boundary. When $K$ is the unit interval this construction produces the unit circle. The next simplest example is the double cover of the Sierpiński gasket, where one considers two copies of the fractal with corresponding boundary points paired. One can extend the definition of energy and Laplacian from $K$ to a fractafold based on $K$. An explicit description of the spectral resolution of the fractafold Laplacian for certain infinite fractafolds is given in [32].

Products of fractals provide another important class of examples for our results. An important point to keep in mind is that the product of p.c.f. fractals is not a p.c.f. fractal. Strichartz described in [37] how one can extend the definition of the Laplacian and energy to products of fractals.

The estimates (2.2) are known to be true for $0<t<1$ on a large number of p.c.f. fractals ([12], [4], [14], [15], [40]) and Sierpiński carpets ([2], [3]), with $R$ being the resistance metric, the constant $d$ being the Hausdorff dimension with respect to the resistance metric, and $\gamma$ a constant specific to the fractal. For the Dirichlet Laplacian on a p.c.f. fractal the estimate for $t>1$ is immediate because the heat kernel decays exponentially at a rate determined by the smallest eigenvalue. In the case of the Neumann Laplacian one should instead modify the heat kernel by subtracting its projection onto the zero eigenspace of constant functions, which has 
exponential decay controlled by the first non-zero eigenvalue. This latter change does not affect the definition of pseudo-differential operators because they act on the eigenspaces with non-zero eigenvalues; accordingly we will abuse notation and refer to this modified heat kernel as just the heat kernel. No modification is needed in the case of blow-ups of p.c.f. fractals. For many fractals, lower bounds on the heat kernel are also known, but we will not need these in this paper. We also note that the heat kernel is holomorphic on $\{\operatorname{Re} z>0\}$ (see [21]).

We mention that estimates of the form (2.2) are also known for sums of even powers of vector fields on homogeneous groups [16]. However, we will not pursue these spaces in this paper.

In this paper we write $A(y) \lesssim B(y)$ if there is a constant $C$ independent of $y$, but which might depend on the space $X$, such that $A(y) \leq C B(y)$ for all $y$. We write $A(y) \sim B(y)$ if $A(y) \lesssim B(y)$ and $B(y) \lesssim A(y)$. If $f(x, y)$ is a function on $X_{1} \times X_{2}$, then we write $\Delta_{1} f$ to denote the Laplacian of $f$ with respect to the first variable and $\Delta_{2} f$ to denote the Laplacian of $f$ with respect to the second variable; repeated subscripts indicate composition, for example $\Delta_{21}=\Delta_{2} \circ \Delta_{1}$. We say that a function $u$ is smooth if $u \in \operatorname{Dom} \Delta^{n}$ for all $n \geq 0$.

\section{Symbols and pseudo-differential operators on fractals}

In this section $(X, R)$ is a metric measure space with a Laplacian $\Delta$ that satisfies the conditions (2.1) and (2.2). We write $P(\lambda)$ for the spectral resolution of the positive self-adjoint operator $-\Delta$. Examples of spaces for which the results of this section apply include compact or infinite blow-ups without boundary or products of copies of the same fractafold. In the case that $X$ is either a compact fractafold without boundary or an infinite fractafold without boundary for which the Laplacian has pure point spectrum ([42], [27]), we write $P_{\lambda}$ for the spectral projection corresponding to the eigenvalue $\lambda$. If $X$ is compact or $\Delta$ has pure point spectrum we fix an orthonormal basis $\left\{\phi_{n}\right\}_{n \in \mathbb{N}}$ or $\left\{\phi_{n}\right\}_{n \in \mathbb{Z}}$ of $L^{2}(\mu)$ consisting of eigenfunctions with compact support and write $\mathcal{D}$ for the dense set of finite linear combinations with respect to this orthonormal basis. If $X$ is a product of such fractals then there is a natural basis of $L^{2}(\mu)$ obtained by taking products of eigenfunctions on each fiber ([37]). The following are the main objects of study in this paper.

Definition 3.1. For fixed $m \in \mathbb{R}$ we define the symbol class $S^{m}$ to be the set of $p \in C^{\infty}((0, \infty))$ with the property that for any integer $k \geq 0$ there is $C_{k}>0$ such that

$$
\left|\left(\lambda \frac{d}{d \lambda}\right)^{k} p(\lambda)\right| \leq C_{k}(1+\lambda)^{\frac{m}{d+1}}
$$

for all $\lambda>0$, where $d$ is an in (2.2).

Remark 3.2. The rationale for dividing $m$ by $d+1$ is that the Laplacian behaves like an operator of order $d+1$. 
If $p$ is any bounded Borel function on $(0, \infty)$ then one can define an operator $p(-\Delta)$ via

$$
p(-\Delta) u=\int_{0}^{\infty} p(\lambda) d P(\lambda)(u) .
$$

This operator extends to a bounded operator on $L^{2}(\mu)$ by the spectral theorem. If $p \in S^{m}$ with $m>0$, then $q(\lambda):=(1+\lambda)^{-m /(d+1)} p(\lambda)$ is bounded and one can define $p(-\Delta)=(I-\Delta)^{-m /(d+1)} q(-\Delta)$.

Definition 3.3. For fixed $m \in \mathbb{R}$ define the class $\Psi D O_{m}$ of pseudo-differential operators on $X$ to be the collection of operators $p(-\Delta)$ with $p \in S^{m}$.

If $X$ is a compact fractafold without boundary or $\Delta$ has pure point spectrum then the formula for a pseudo-differential operator is

$$
p(-\Delta) u=\sum_{\lambda \in \Lambda} p(\lambda) P_{\lambda} u
$$

for $p \in S^{m}$ and $u \in \mathcal{D}$, where $\Lambda$ is the spectrum of $-\Delta$.

Proposition 3.4 (Symbolic calculus). If $p_{1} \in S^{m_{1}}$ and $p_{2} \in S^{m_{2}}$ then $p_{1} p_{2} \in$ $S^{m_{1}+m_{2}}$ and

$$
p_{1}(-\Delta) \circ p_{2}(-\Delta)=p_{1} p_{2}(-\Delta)
$$

Proof. Let $k \geq 1$. Using Leibniz's formula we have that

$$
\lambda^{k} \frac{d^{k}}{d \lambda^{k}}\left(p_{1} p_{2}\right)(\lambda)=\sum_{j=0}^{k}\left(\begin{array}{c}
k \\
j
\end{array}\right) \lambda^{j} \frac{d^{j}}{d \lambda^{j}} p_{1}(\lambda) \lambda^{k-j} \frac{d^{k-j}}{d \lambda^{k-j}} p_{2}(\lambda)
$$

Therefore

$$
\left|\lambda^{k} \frac{d^{k}}{d \lambda^{k}}\left(p_{1} p_{2}\right)(\lambda)\right| \leq \sum_{j=0}^{k}\left(\begin{array}{l}
k \\
j
\end{array}\right)(1+\lambda)^{\frac{m_{1}}{d+1}}(1+\lambda)^{\frac{m_{2}}{d+1}}=2^{k}(1+\lambda)^{\frac{m_{1}+m_{2}}{d+1}} .
$$

Thus $p_{1} p_{2} \in S^{m_{1}+m_{2}}$. Using the identity $P(\lambda)(p(-\Delta) u)=p(\lambda) P(\lambda)(u)$ we have that

$$
\begin{aligned}
p_{1}(-\Delta) \circ p_{2}(-\Delta) u & =\int p_{1}(\lambda) P(\lambda)\left(p_{2}(-\Delta) u\right) \\
& =\int p_{1}(\lambda) p_{2}(\lambda) P(\lambda)(u)=\left(p_{1} p_{2}\right)(-\Delta) u
\end{aligned}
$$

The main result of this section is Theorem 3.6, which says that if $p \in S^{0}$ then $p(-\Delta)$ is given by integration with respect to a kernel that is smooth off the diagonal and satisfies specific decay estimates off the diagonal. When $X$ is a fractafold based on a p.c.f. fractal we then obtain from Theorem 1 in [19] that $p(-\Delta)$ is a Calderón-Zygmund operator in the sense of Section I.6.5 in [31]. We begin with a technical lemma. 
Lemma 3.5. Let $\alpha>0$ and $R>0$ be fixed. Then

$$
\sum_{n \in \mathbb{Z}} 2^{\frac{n \alpha}{d+1}} \int \exp \left(-c R^{(d+1) \gamma} 2^{n \gamma}\left(1+\xi^{2}\right)^{-\frac{\gamma+1}{2}}\right) \frac{1}{\left(1+\xi^{2}\right)^{j}} d \xi \leq C R^{-\alpha}
$$

provided $j \geq \frac{\alpha(\gamma+1)}{2 \gamma(d+1)}+\frac{1}{2}$.

Proof. Consider the integral

$$
\begin{aligned}
I & =\int_{\mathbb{R}} \exp \left(-A\left(1+\xi^{2}\right)^{-\frac{\gamma+1}{2}}\right) \frac{1}{\left(1+\xi^{2}\right)^{j}} d \xi \\
& =2 \int_{0}^{\infty} \exp \left(-A\left(1+\xi^{2}\right)^{-\frac{\gamma+1}{2}}\right) \frac{1}{\left(1+\xi^{2}\right)^{j}} d \xi .
\end{aligned}
$$

Since $2\left(1+\xi^{2}\right) \geq(1+\xi)^{2}$ on $(0, \infty)$ we have

$$
I \lesssim \int_{1}^{\infty} \exp \left(-\frac{A}{2} \xi^{-\gamma+1}\right) \frac{d \xi}{\xi^{2 j}} \simeq A^{\frac{1-2 j}{\gamma+1}} \int_{0}^{A / 2} e^{-t} t^{\frac{2 j-1}{\gamma+1}} \frac{d t}{t} \leq C(j, \gamma) A^{\frac{1-2 j}{\gamma+1}}
$$

for $j>1 / 2$. We use this bound for $A \geq 1$ and the obvious bound by $C(j)$ for $A \leq 1$. Let $A=c R^{(d+1) \gamma} 2^{n \gamma}$ and $n_{0}$ be such that $A \geq 1$ if and only if $n \geq n_{0}$. The series in the statement of the lemma is bounded by

$$
C(j) \sum_{n<n_{0}} 2^{\frac{n \alpha}{d+1}}+C(j, \gamma) R^{\frac{(d+1)(1-2 j) \gamma}{\gamma+1}} \sum_{n \geq n_{0}} 2^{n\left(\frac{\alpha}{d+1}-\frac{(2 j-1) \gamma}{\gamma+1}\right)},
$$

which converges for $j$ as in the lemma. The estimate follows from the fact that $2^{n_{0}} \simeq c^{-1} R^{-(d+1)}$.

Theorem 3.6. Let $p:(0, \infty) \rightarrow \mathbb{C}$ be an $S^{0}$-symbol, that is $p$ is smooth and for all $k \geq 0$ there is $C_{k}>0$ such that

$$
\left|\lambda^{k} \frac{\partial^{k}}{\partial \lambda^{k}} p(\lambda)\right| \leq C_{k}
$$

Then $p(-\Delta)$ has a kernel $K(x, y)$ that is smooth off the diagonal of $X \times X$ and satisfies

$$
|K(x, y)| \lesssim R(x, y)^{-d}
$$

and

$$
\left|\Delta_{x}^{l} \Delta_{y}^{k} K(x, y)\right| \lesssim R(x, y)^{-d-(l+k)(d+1)} .
$$

Proof. We begin with the Littlewood-Paley dyadic decomposition from [31] (see page 242). Let $\eta$ be a $C^{\infty}$ function with $\eta(\lambda)=1$ if $|\lambda| \leq 1$ and $\eta(\lambda)=0$ if $|\lambda| \geq 2$ and let $\delta(\lambda)=\eta(\lambda)-\eta(2 \lambda)$. Then $\operatorname{supp} \delta \subseteq\{1 / 2 \leq|\lambda| \leq 2\}$ and

$$
\sum_{n \in \mathbb{Z}} \delta\left(2^{-n} \lambda\right)=1
$$


where for each $\lambda$ there are only two nonzero terms in the above sum. Then $\delta$ is $C_{c}^{\infty}$ and we let $D_{k}>0$ for each $k \geq 0$ such that

$$
\left|\frac{d^{k}}{d \lambda^{k}} \delta(\lambda)\right| \leq D_{k}
$$

For $n \in \mathbb{Z}$ let $p_{n}(\lambda)=p(\lambda) \delta\left(2^{-n} \lambda\right)$. Then $\operatorname{supp} p_{n} \subseteq\left[2^{n-1}, 2^{n+1}\right]$ (since we assume that $p$ is defined on $(0, \infty))$ and

$$
p(\lambda)=\sum_{n \in \mathbb{Z}} p_{n}(\lambda)
$$

Moreover we can use (3.2) and the fact that the support of $p_{n}$ has support in $\left[2^{n-1}, 2^{n+1}\right]$ to bound $\frac{d^{k}}{d \lambda^{k}} p_{n}$ by a constant that depends only on $k$ :

$$
\begin{aligned}
\left|\frac{d^{k}}{d \lambda^{k}} p_{n}(\lambda)\right| & =\left|\sum_{j=1}^{k}\left(\begin{array}{c}
k \\
j
\end{array}\right) \frac{d^{j}}{d \lambda^{j}} p(\lambda) \cdot 2^{-n(k-j)} \frac{d^{k-j}}{d \lambda^{k-j}} \delta\left(2^{-n} \lambda\right)\right| \\
& \leq 2^{-n k} \sum_{j=0}^{k}\left(\begin{array}{c}
k \\
j
\end{array}\right) \frac{C_{j}}{\lambda^{j}} D_{k-j} 2^{n j} \leq 2^{-n k} C(k) \sum_{j=1}^{k} \frac{2^{j}}{2^{n j}} 2^{n j}=\bar{C}_{k} 2^{-n k},
\end{aligned}
$$

where $C(k)=\max C_{j} D_{j-k}$ and $\bar{C}_{k}=2^{k+1} C(k)$.

Fix now $n \in \mathbb{Z}$ and set $f_{n}(\lambda)=p_{n}\left(2^{n} \lambda\right) e^{\lambda}$. Then supp $f_{n} \subseteq[1 / 2,2]$ and for all $k \geq 0$ we have that

$$
\frac{d^{k}}{d \lambda^{k}} f_{n}(\lambda)=\sum_{j=0}^{k}\left(\begin{array}{l}
k \\
j
\end{array}\right) 2^{n j} \frac{d^{j}}{d \lambda^{j}} p_{n}\left(2^{n} \lambda\right) e^{\lambda} .
$$

Using (3.7) and the fact that $\lambda \leq 2$ we obtain

$$
\left|\frac{d^{k}}{d \lambda^{k}} f_{n}(\lambda)\right| \leq \sum_{j=0}^{k}\left(\begin{array}{l}
k \\
j
\end{array}\right) 2^{n j} \bar{C}_{j} 2^{-n j} e^{2}=: A_{k} .
$$

It follows immediately that there are constants $B_{k}$ independent of $n$ such that the Fourier transform $\hat{f}_{n}(\xi)$ of $f_{n}$ satisfies $\left|\xi^{k} \hat{f}_{n}(\xi)\right| \leq B_{k}$, and thus for each $k \in \mathbb{Z}$

$$
\left|\hat{f}_{n}(\xi)\right| \leq \frac{\bar{D}_{k}}{\left(1+\xi^{2}\right)^{k}} .
$$

for some constants $\bar{D}_{k}$ independent of $n$.

Now, by Fourier inversion,

$$
p_{n}(\lambda)=e^{-\lambda 2^{-n}} f_{n}\left(\lambda 2^{-n}\right)=e^{-\lambda 2^{-n}} \frac{1}{2 \pi} \int \hat{f}_{n}(\xi) e^{i \lambda \xi 2^{-n}} d \xi,
$$

so that

$$
p_{n}(-\Delta) u=\frac{1}{2 \pi} \int \hat{f}_{n}(\xi) e^{\Delta(1-i \xi) 2^{-n}} u d \xi
$$


and the kernel of $p_{n}(-\Delta)$ is given by

$$
K_{n}(x, y)=\frac{1}{2 \pi} \int \hat{f}_{n}(\xi) h_{2^{-n}-i \xi 2^{-n}}(x, y) d \xi
$$

where $h_{z}(x, y)$ is the complex heat kernel, which is holomorphic on $\{\operatorname{Re} z>0\}$. A proof identical with that of Lemma 3.4.6 of [8] shows that there is $C>0$ such that

$$
\left|h_{z}(x, y)\right| \leq C(\operatorname{Re} z)^{-\frac{d}{d+1}} .
$$

Using the above estimate together with the heat kernel estimates (2.2), Lemma 9 of [9] implies that

$$
\left|h_{z}(x, y)\right| \leq C 2^{\frac{d}{d+1}}(|z| \cos (\theta))^{-\frac{d}{d+1}} \exp \left(-\frac{c \gamma}{2} R(x, y)^{(d+1) \gamma}|z|^{-\gamma} \cos (\theta)\right)
$$

where $z=r(\cos \theta+i \sin \theta)$. For $z=2^{-n}-i \xi 2^{-n}$ we have that $|z|=2^{-n}\left(1+\xi^{2}\right)^{1 / 2}$, $\cos \theta=\left(1+\xi^{2}\right)^{-1 / 2}$, and $|z| \cos \theta=2^{-n}$. Thus

$$
\left|h_{2^{-n}-i \xi 2^{-n}}(x, y)\right| \leq C 2^{\frac{d}{d+1}} 2^{\frac{n d}{d+1}} \exp \left(-\frac{c \gamma}{2} R(x, y)^{(d+1) \gamma} 2^{n \gamma}\left(1+\xi^{2}\right)^{-\frac{\gamma+1}{2}}\right)
$$

and

$$
\left|K_{n}(x, y)\right| \leq C(d, j) 2^{\frac{n d}{d+1}} \int \exp \left(-\frac{c \gamma}{2} R(x, y)^{(d+1) \gamma} 2^{n \gamma}\left(1+\xi^{2}\right)^{-\frac{\gamma+1}{2}}\right) \frac{1}{\left(1+\xi^{2}\right)^{j}} d \xi
$$

for all $j \geq 1$, where the constants $C(d, j)$ depend only on $d$ and $j$. Lemma 3.5 with $\alpha=d$ and $R=R(x, y)$ implies that if we choose $j$ large enough then $K(x, y)=$ $\sum_{n \in \mathbb{Z}} K_{n}(x, y)$ is defined and continuous off the diagonal and it satisfies (3.3). Finally, using (3.6), we see that $K(x, y)$ is the kernel of $p(-\Delta)$.

Next we want to prove the $K(x, y)$ is smooth off the diagonal and show that the estimates (3.4) hold. For simplicity we give the complete argument only in the case $l=0$ and $k=1$, i.e., we show that

$$
\left|\Delta_{y} K(x, y)\right| \lesssim R(x, y)^{-2 d-1}
$$

Let $q(\lambda)=\lambda p(\lambda)$ and $q_{n}(\lambda)=\lambda p_{n}(\lambda)$ for all $n \in \mathbb{Z}$. By (3.7) we obtain that $\left|q_{n}^{(k)}(\lambda)\right| \leq C(k) 2^{(1-k) n}$ for all $k \geq 0$. If we set $g_{n}(\lambda)=2^{-n} q_{n}\left(2^{n} \lambda\right) e^{\lambda}$, we then obtain $\left|g_{n}^{(k)}(\lambda)\right| \leq A(k)$, where $A(k)$ are constants independent of $n$. Then a computation similar to $(3.9)$ shows that

$$
\left|\hat{g}_{n}(\xi)\right| \leq \frac{B(k)}{\left(1+\xi^{2}\right)^{k}}
$$

for all $k \geq 0$, with $B(k)$ independent of $n$. Using the Fourier inversion formula we have that

$$
q_{n}(\lambda)=\frac{1}{2 \pi} 2^{n} \int \hat{g}_{n}(\xi) e^{-\lambda\left(2^{-n}-i \xi 2^{-n}\right)} d \xi
$$


and a similar formula holds for $q_{n}(-\Delta)$. Hence

$$
\Delta_{y} K_{n}(x, y)=\frac{1}{2 \pi} 2^{n} \int \hat{g}_{n}(\xi) h_{2^{-n}-i \xi 2^{-n}}(x, y) d \xi
$$

and so

$$
\begin{aligned}
\left|\Delta_{y} K_{n}(x, y)\right| & \leq C(d) 2^{n} 2^{\frac{n d}{d+1}} \int\left|\hat{g}_{n}(\xi)\right| \exp \left(-c(\gamma) \frac{R(x, y)^{(d+1) \gamma} 2^{n \gamma}}{\left(1+\xi^{2}\right)^{\frac{\gamma+1}{2}}}\right) d \xi \\
& \leq C(d, j) 2^{\frac{n(2 d+1)}{d+1}} \int \exp \left(-c(\gamma) \frac{R(x, y)^{(d+1) \gamma} 2^{n \gamma}}{\left(1+\xi^{2}\right)^{\frac{\gamma+1}{2}}}\right) \frac{1}{\left(1+\xi^{2}\right)^{j}} d \xi
\end{aligned}
$$

for all $j \geq 1$. Using Lemma 3.5 with $\alpha=2 d+1$ we find for $j$ large enough that $\Delta_{y} K(x, y)=\sum_{n} \Delta_{y} K_{n}(x, y)$ is well defined and continuous off the diagonal and it satisfies (3.15).

To prove (3.4) for general $l$ and $k$, one can repeat the above steps for the functions $q(\lambda)=\lambda^{l+k} p(\lambda), q_{n}(\lambda)=\lambda^{l+k} p_{n}(\lambda)$, and $g_{n}(\lambda)=2^{-n(l+k)} q_{n}(\lambda) e^{\lambda}$ and apply Lemma 3.5 with $\alpha=d+(l+k)(d+1)$.

Corollary 3.7. Assume that $X$ is a fractafold without boundary or a product of such fractafolds. If $p \in S^{0}$ then $p(-\Delta)$ is a Calderón-Zygmund operator and, thus, it extends to a bounded operator on $L^{q}(\mu)$ for all $1<q<\infty$ and satisfies weak 1-1 estimates.

Proof. Using the estimates (3.3) and (3.4) (with $l=0$ and $k=1$ ), Theorem 1.1 of [19] implies that $p(-\Delta)$ is a Calderón-Zygmund operator.

Remark 3.8. The boundedness of $p(-\Delta)$ on $L^{q}(\mu), 1<q<\infty$, can be obtained also using the results of [11].

Corollary 3.9. If $p \in S^{m}$ then $p(-\Delta)$ is given by integration with respect to a kernel $K_{p}$ that is smooth off the diagonal.

Proof. If $p \in S^{m}$ then $q(\lambda)=p(\lambda)(1+\lambda)^{-m} \in S^{0}$ and Theorem 3.6 implies that $q(-\Delta)$ has a kernel $K_{q}$ that is smooth off the diagonal. Then

$$
K_{p}(x, y)=\left(I-\Delta_{x}\right)^{m} K_{q}(x, y)
$$

is smooth off the diagonal and it is the kernel of $p(-\Delta)$.

As a consequence, we obtain that, for $\operatorname{Re} s \geq 0$, the Bessel potentials

$$
(I-\Delta)^{-s} u=\sum_{\lambda \in \Lambda}(1+\lambda)^{-s} P_{\lambda} u
$$

and the Riesz potentials

$$
(-\Delta)^{-s} u=\sum_{\lambda \in \Lambda} \lambda^{-s} P_{\lambda} u
$$

have smooth kernels and are bounded on $L^{p}(\mu)$, for $1<p<\infty$, with operator norm of at most polynomial growth in $\operatorname{Im} s$ (when $\operatorname{Re} s=0$ ). These facts were previously proved in [19]. 


\section{Sobolev spaces}

In this section we assume that $X$ is a compact fractafold without boundary based on a p.c.f. fractal $K$, an infinite blow-up of $K$ without boundary, or a product of copies of the same fractafold without boundary. Moreover, we assume that if $X$ is non-compact then $\Delta$ has pure point spectrum. Therefore we can consider that $L^{2}(\mu)$ is spanned by compactly supported eigenfunctions of $-\Delta$. As before, we fix an orthonormal basis $\left\{\phi_{n}\right\}$ of $L^{2}(\mu)$ consisting of such eigenfunctions and we write $\mathcal{D}$ for the space of finite linear combinations of $\phi_{n}$ 's.

Definition 4.1 ( $L^{2}$-Sobolev spaces). For $s \geq 0$ and $u \in L^{2}$ define

$$
\|u\|_{H^{s}}^{2}:=\sum_{\lambda \in \Lambda}(1+\lambda)^{\frac{2 s}{d+1}}\left\|P_{\lambda} u\right\|_{2}^{2} .
$$

We say that $u \in H^{s}$ if and only if $\|u\|_{H^{s}}<\infty$.

For $s \geq 0$ if $u \in H^{s}$ then $u \in L^{2}(\mu)$. So for $s \geq 0$ there is no harm in starting with $u \in L^{2}(\mu)$ in Definition 4.1. Moreover if $s_{1} \leq s_{2}$ then $H^{s_{2}} \subseteq H^{s_{1}}$.

For $s<0$ we define $H^{s}$ using the distribution theory developed in [25]. Namely, for $s>0, H^{-s}$ is the dual of $H^{s}$ via

$$
\langle f, \varphi\rangle=\sum\left\langle f, \phi_{n}\right\rangle \overline{\left\langle\varphi, \phi_{n}\right\rangle}
$$

for $f \in H^{-s}$ and $\varphi \in H^{s}$. The fact that the above linear functional is bounded is a consequence of the Cauchy-Schwarz inequality:

$$
\langle f, \varphi\rangle=\sum_{n} \lambda_{n}^{\frac{-s}{d+1}}\left\langle f, \phi_{n}\right\rangle \lambda_{n}^{\frac{s}{d+1}} \overline{\left\langle\varphi, \phi_{n}\right\rangle} \leq\|f\|_{H^{-s}}\|\varphi\|_{H^{s}}
$$

Lemma 4.2. For $s$ a positive integer, $u \in H^{s(d+1)}$ if and only if $(I-\Delta)^{k} u \in L^{2}(\mu)$ for all $k \leq s$.

Proof. Notice that

$$
\|u\|_{H^{s(d+1)}}^{2}=\sum_{\lambda \in \Lambda}(1+\lambda)^{2 s}\left\|P_{\lambda} u\right\|_{2}^{2}=\left\|(I-\Delta)^{s} u\right\|_{2}^{2} .
$$

From this equality the statement follows.

Proposition 4.3. If $p \in S^{m}$ then $p(-\Delta): H^{s} \rightarrow H^{s-m}$ for all $s$ and $m$.

Proof. Recall that $P_{\lambda}(p(-\Delta) u)=p(\lambda) P_{\lambda} u$. Then

$$
\begin{aligned}
& \|p(-\Delta) u\|_{H^{s-m}}^{2}=\sum_{\lambda \in \Lambda}(1+\lambda)^{\frac{2(s-m)}{d+1}}\left\|P_{\lambda}(p(-\Delta) u)\right\|_{2}^{2} \\
& =\sum_{\lambda \in \Lambda}(1+\lambda)^{\frac{2(s-m)}{d+1}}|p(\lambda)|^{2}\left\|P_{\lambda} u\right\|_{2}^{2} \lesssim \sum_{\lambda \in \Lambda}(1+\lambda)^{\frac{2 s}{d+1}}\left\|P_{\lambda} u\right\|_{2}^{2}=\|u\|_{H^{s}}^{2} .
\end{aligned}
$$

Thus $p(-\Delta) u \in H^{s-m}$ whenever $u \in H^{s}$. 
Proposition 4.4. For $s \geq 0, H^{s}$ equals the image of $L^{2}(\mu)$ under $(I-\Delta)^{-s /(d+1)}$.

Proof. Let $u, f \in L^{2}(\mu)$ such that $u=(I-\Delta)^{-s /(d+1)} f$. Then

$$
P_{\lambda} u=\frac{1}{(1+\lambda)^{s /(d+1)}} P_{\lambda} f
$$

Therefore,

$$
\|u\|_{H^{s}}^{2}=\sum_{\lambda \in \Lambda}(1+\lambda)^{\frac{2 s}{d+1}} \frac{1}{(1+\lambda)^{2 s /(d+1)}}\left\|P_{f} f\right\|_{2}^{2}=\|f\|_{2}^{2}<\infty .
$$

Now let $u \in H^{s}$ and define $f=(I-\Delta)^{s /(d+1)} u$, that is,

$$
f=\sum(1+\lambda)^{\frac{s}{d+1}} P_{\lambda} u .
$$

Then

$$
\|f\|_{2}^{2}=\sum_{\lambda \in \Lambda}(1+\lambda)^{\frac{2 s}{d+1}}\left\|P_{\lambda} u\right\|_{2}^{2}=\|u\|_{H^{s}}^{2}<\infty .
$$

Hence $f \in L^{2}(\mu)$ and $u=(I-\Delta)^{-s /(d+1)} f$.

We extend next the definition of Sobolev spaces to $L^{p}$ spaces, using Proposition 4.4 as the starting point. Namely, we are going to replace $L^{2}(\mu)$ by $L^{p}(\mu)([30])$.

Definition 4.5. We define the $L^{p}$ Sobolev space $L_{s}^{p}$ for $s \geq 0$ and $1<p<\infty$ to be the image of $L^{p}$ under $(I-\Delta)^{-s /(d+1)}$ with the norm

$$
\left\|(I-\Delta)^{-s /(d+1)} f\right\|_{L_{s}^{p}}=\|f\|_{L^{p}(\mu)} .
$$

Note that by Corollary 3.7 we may regard $L_{s}^{p}$ as a closed subspace of $L^{p}$, with $\|u\|_{p} \leq c\|u\|_{L_{s}^{p}}$. For $s=0$ we have $L_{0}^{p}=L^{p}$ and for $p=2$ we have $L_{s}^{2}=H^{s}$.

Proposition 4.6. If $0<s_{0} \leq s_{1}<\infty$ and $1<p_{0}, p_{1}<\infty$ then the complex interpolation space $\left[L_{s_{0}}^{p_{0}}, L_{s_{1}}^{p_{1}}\right]_{\theta}$ may be identified with $L_{s}^{p}$ where $0<\theta<1, s=$ $(1-\theta) s_{0}+\theta s_{1}$, and $1 / p=(1-\theta) / p_{0}+\theta / p_{1}$.

Proof. The proof is as in the Euclidean case, using the comments following Proposition 3.7 .

Proposition 4.7. If $p \in S^{m}$ then $p(-\Delta)$ is a bounded operator from $L_{s}^{p}$ into $L_{s-m}^{p}$.

Proof. Let $f \in L_{s}^{p}$. Then there is $g \in L^{p}$ such that $f=(I-\Delta)^{-s /(d+1)} g$. Then $p(-\Delta) f=p(-\Delta)(I-\Delta)^{-s /(d+1)} g$. By Proposition 3.4 and Corollary 3.7

$$
(I-\Delta)^{(s-m) /(d+1)} p(-\Delta)(I-\Delta)^{-s /(d+1)} g \in L^{p} .
$$

This is equivalent to $p(-\Delta) f=(I-\Delta)^{-(s-m) /(d+1)} g \in L_{s-m}^{p}$.

Lemma 4.8. The Sobolev space $L_{d+1}^{p}$ equals $\operatorname{dom}_{L^{p}}(\Delta)$.

Proof. Let $u \in \operatorname{dom}_{L^{p}}(\Delta)$. Then $u \in \operatorname{dom}(\mathcal{E})$ and there is $f \in L^{p}$ such that $f=$ $-\Delta u$. That is,

$$
\int f v d \mu=\mathcal{E}(u, v) \text { for all } v \in \operatorname{dom}(\mathcal{E})
$$


If $v=\phi_{\lambda}$, where $\phi_{\lambda}$ is any eigenfunction corresponding to $\lambda$, then $\lambda \int u \phi_{\lambda} d \mu=$ $\int f \phi_{\lambda} d \mu$. This implies that $(I-\Delta)^{-1}(f+u)=u$.

For the converse, notice first that if $\lambda_{1}, \lambda_{2} \in \Lambda$ and $\phi_{\lambda_{i}}$ is any eigenfunction corresponding to $\lambda_{i}, i=1,2$, then $\mathcal{E}\left(\phi_{\lambda_{1}}, \phi_{\lambda_{2}}\right)=\lambda_{1} \delta\left(\lambda_{1}, \lambda_{2}\right)$. Let $u=(I-\Delta)^{-1} g$ for some $g \in L^{p}$. Set $f=g-u \in L^{p}$. For any $\lambda \in \Lambda$ we have

$$
\int f \phi_{\lambda} d \mu=\int(g-u) \phi_{\lambda} d \mu=\frac{\lambda}{\lambda+1} \int g \phi_{\lambda} d \mu \text {. }
$$

Also

$$
\mathcal{E}\left(u, \phi_{\lambda}\right)=\frac{1}{1+\lambda} \int g \phi_{\lambda} d \mu \mathcal{E}\left(\phi_{\lambda}, \phi_{\lambda}\right)=\frac{\lambda}{1+\lambda} \int g \phi_{\lambda} d \mu .
$$

It follows by linearity and density that $\int f v d \mu=\mathcal{E}(u, v)$ for all $v \in \operatorname{dom}(\mathcal{E})$. Thus $-\Delta u=f$.

Theorem 4.9 (Sobolev embedding theorem). If $s<\frac{d}{p}$ then $L_{s}^{p} \subseteq L^{q}$ for $\frac{1}{q}=\frac{1}{p}-\frac{s}{d}$.

Proof. We have all the ingredients needed to use the same proof as in Theorem 3.11 of $[36]$.

\section{Pseudo-differential operators on products of fractals}

In this section we extend the definition of pseudo-differential operators to products of metric measure spaces that satisfy the hypotheses of Section 2 . Let $N \geq 2$ be fixed and let $\left(X_{1}, R_{1}\right), \ldots,\left(X_{N}, R_{N}\right)$ be $N$ metric spaces such that $X_{i}$ has measure $\mu_{i}$ and Laplacian $\Delta_{i}$. In the case that $X_{i}$ is a fractafold, then we take $\Delta_{i}$ as defined using a self-similar Dirichlet energy $\mathcal{E}_{i}$. We assume that the heat kernel $h^{(i)}$ associated with $\Delta_{i}$ satisfies the estimates (2.2) with $d=d_{i}, \gamma=\gamma_{i}, R=R_{i}$, for all $i=1, \ldots, N$. Let $X=X_{1} \times \cdots \times X_{N}$ be the product space and let $\mu=\mu_{1} \times \cdots \times \mu_{N}$ be the product measure on $X$. We write $x=\left(x_{1}, \ldots, x_{n}\right)$ for elements in $X$, $\lambda=\left(\lambda_{1}, \ldots, \lambda_{N}\right)$ for elements in $(0, \infty)^{N}$ and $\Delta=\left(\Delta_{1}, \ldots, \Delta_{N}\right)$. Recall that there is a unique spectral decomposition $P_{\lambda}$ such that

$$
P_{\lambda} u_{1} \times u_{2} \times \cdots \times u_{N}=P_{\lambda_{1}} \otimes P_{\lambda_{2}} \otimes \cdots \otimes P_{\lambda_{N}} u_{1} \times \cdots \times u_{N},
$$

where $u_{1} \times \cdots \times u_{n}(x)=u_{1}\left(x_{1}\right) u_{2}\left(x_{2}\right) \ldots u_{N}\left(x_{N}\right)$,

$P_{\lambda_{1}} \otimes P_{\lambda_{2}} \otimes \cdots \otimes P_{\lambda_{N}} u_{1}\left(x_{1}\right) u_{2}\left(x_{2}\right) \ldots u_{N}\left(x_{N}\right)=P_{\lambda_{1}} u_{1}\left(x_{1}\right) P_{\lambda_{2}} u_{2}\left(x_{2}\right) \ldots P_{\lambda_{N}} u_{N}\left(x_{N}\right)$,

and $P_{\lambda_{i}}$ is the spectral projection corresponding to the eigenvalue $\lambda_{i}$ of $-\Delta_{i}$.

Recall from [37] that if $\left\{\phi_{n}^{i}\right\}$ is an orthonormal basis for $L^{2}\left(\mu_{i}\right)$ consisting of compactly supported eigenfunctions of $-\Delta_{i}$, then

$$
\phi_{k_{1}, \ldots, k_{N}}(x)=\phi_{k_{1}}^{1}\left(x_{1}\right) \cdots \phi_{k_{N}}^{N}\left(x_{N}\right),
$$

where $k_{i} \in \mathbb{Z}$ for all $i=1, \ldots, N$, form an orthonormal basis for $L^{2}(\mu)$. In this case we write $\mathcal{D}^{N}$ for the set of finite linear combinations of the $\phi_{k_{1}, \ldots, k_{N}}$. 
Definition 5.1. For $m \in \mathbb{Z}$ we define the symbol class $S^{m}$ on $X$ to consist of the set of smooth functions $p:(0, \infty)^{N} \rightarrow \mathbb{C}$ that satisfy the property that for every $\alpha=\left(\alpha_{1}, \ldots, \alpha_{N}\right) \in \mathbb{N}^{N}$ there is a positive constant $C_{\alpha}$ such that

$$
\lambda^{\alpha}\left|\frac{\partial^{\alpha}}{\partial \lambda^{\alpha}} p(\lambda)\right| \leq C_{\alpha}(1+\lambda)^{\frac{m}{d+1}},
$$

where $\lambda^{\alpha}=\lambda_{1}^{\alpha_{1}} \lambda_{2}^{\alpha_{2}} \cdots \lambda_{N}^{\alpha_{N}}$, and

$$
\frac{\partial^{\alpha}}{\partial \lambda^{\alpha}} p(\lambda)=\frac{\partial^{\alpha_{1}}}{\partial \lambda_{1}^{\alpha_{1}}} \cdots \frac{\partial^{\alpha_{N}}}{\partial \lambda_{N}^{\alpha_{N}}} p\left(\lambda_{1}, \ldots, \lambda_{N}\right) .
$$

Remark 5.2. Note that each $\lambda_{i}$ is positive and we omit the absolute values of $\lambda$ in the above definition. Notice also that if $p$ satisfies the condition (5.2) then it also satisfies the condition

$$
|\lambda|^{|\alpha|}\left|\frac{\partial^{\alpha}}{\partial \lambda^{\alpha}} p(\lambda)\right| \leq C_{\alpha}(1+\lambda)^{\frac{m}{d+1}},
$$

where $|\lambda|=\left(\lambda_{1}^{2}+\cdots+\lambda_{N}^{2}\right)^{1 / 2}$ if $\lambda \in(0, \infty)^{N}$, and $|\alpha|=\alpha_{1}+\cdots+\alpha_{N}$ if $\alpha \in \mathbb{N}^{N}$. Condition (5.2) is usually called the Marcinkiewicz condition while (5.3) is the Hörmander condition. Many authors define pseudo-differential operators on Euclidean spaces or manifolds using the Hörmander condition. We chose to use Marcinkiewicz condition in the definition because it is more general and it makes the proof of the main theorem in this section more transparent.

If $p:(0, \infty)^{N} \rightarrow \mathbb{C}$ is a bounded Borel function then we can define an operator $p(-\Delta)$ acting on $L^{2}(\mu)$ via

$$
p(-\Delta) u=\int_{(0, \infty)^{N}} p(\lambda) P_{\lambda}(u),
$$

where $P_{\lambda}$ is defined as in (5.1).

Definition 5.3. For $m \in \mathbb{Z}$ define the class $\Psi D O_{m}^{N}$ of pseudo-differential operators on $X$ to be the collection of operators $p(-\Delta)$ with $p \in S^{m}$.

The spectral theorem implies that, if $m=0$, then $p(-\Delta)$ extends to $L^{2}(\mu)$. Moreover we will show that, as in the single variable case, these operators are given by integration with respect to kernels that are smooth off the diagonal and, if $X$ is a product of fractafolds, they are Calderón-Zygmund operators on $X$. The following is the main theorem of this section.

Theorem 5.4. Suppose that $p \in S^{0}$, that is, $p$ is smooth and

$$
\lambda^{\alpha}\left|\frac{\partial^{\alpha}}{\partial \lambda^{\alpha}} p(\lambda)\right| \leq C_{\alpha}
$$

for all $\alpha=\left(\alpha_{1}, \ldots, \alpha_{N}\right) \in \mathbb{N}^{N}$. Then $p(-\Delta)$, where $\Delta=\left(\Delta_{1}, \ldots, \Delta_{N}\right)$, is given by integration with respect to a kernel $K_{p}$ that is smooth off the diagonal and satisfies

$$
\left|K_{p}(x, y)\right| \lesssim \prod_{k=1}^{N} R_{k}\left(x_{k}, y_{k}\right)^{-d_{k}}
$$


and

$$
\left|\Delta_{x, i}^{\beta_{1}} \Delta_{y, j}^{\beta_{2}} K_{p}(x, y)\right| \lesssim R_{i}\left(x_{i} y_{i}\right)^{-\beta_{1}\left(d_{i}+1\right)} R_{j}\left(x_{j}, y_{j}\right)^{-\beta_{2}\left(d_{j}+1\right)} \prod_{k=1}^{N} R\left(x_{k}, y_{k}\right)^{-d_{k}}
$$

for all $i, j=1, \ldots, N$, where $x=\left(x_{1}, \ldots, x_{N}\right), y=\left(y_{1}, \ldots, y_{N}\right)$ and $\Delta_{x, i}$ is the Laplacian with respect to $x_{i}$ and $\Delta_{y, j}$ is the Laplacian with respect to $y_{j}$.

Proof. For simplicity we prove the result for $N=2$. The difference between this and the general case is a matter of notation. We use the Littlewood-Paley decomposition from [31], page 242, in each variable. Let $\delta$ be the smooth function from the proof of Theorem 3.6 and let $D_{k}$ be the bounds from (3.5). For $n, m \in \mathbb{Z}$ define

$$
p_{n, m}(\lambda)=p(\lambda) \delta\left(2^{-n} \lambda_{1}\right) \delta\left(2^{-m} \lambda_{2}\right),
$$

where $\lambda=\left(\lambda_{1}, \lambda_{2}\right) \in(0, \infty)^{2}$. Then $p_{n, m}$ is a smooth function with

$$
\operatorname{supp} p_{n, m} \subseteq\left[2^{n-1}, 2^{n+1}\right] \times\left[2^{m-1}, 2^{m+1}\right] \text { for all } n, m \in \mathbb{Z} \text {. }
$$

Let $n, m \in \mathbb{Z}$ be fixed. We use Leibniz's formula to estimate the bounds on the derivatives of $p_{n, m}$. If $\alpha=\left(\alpha_{1}, \alpha_{2}\right) \in \mathbb{N}^{2}$ then we have

$$
\begin{aligned}
\frac{\partial^{\alpha}}{\partial \lambda^{\alpha}} p_{n, m}(\lambda)= & \frac{\partial^{\alpha_{1}}}{\partial \lambda_{1}^{\alpha_{1}}} \frac{\partial^{\alpha_{2}}}{\partial \lambda_{2}^{\alpha_{2}}}\left(p(\lambda) \delta\left(2^{-n} \lambda_{1}\right) \delta\left(2^{-m} \lambda_{2}\right)\right) \\
= & \frac{\partial^{\alpha_{1}} \delta\left(2^{-n} \lambda_{1}\right)}{\partial \lambda_{1}^{\alpha_{1}}}\left(\sum_{k=0}^{\alpha_{2}}\left(\begin{array}{c}
\alpha_{2} \\
k
\end{array}\right) \frac{\partial^{k} p(\lambda)}{\partial \lambda_{2}^{k}} 2^{-m\left(\alpha_{2}-k\right)} \delta^{\left(\alpha_{2}-k\right)}\left(2^{-m} \lambda_{2}\right)\right) \\
= & \sum_{j=0}^{\alpha_{1}} \sum_{k=0}^{\alpha_{2}}\left[\left(\begin{array}{c}
\alpha_{1} \\
j
\end{array}\right)\left(\begin{array}{c}
\alpha_{2} \\
k
\end{array}\right)\left(\frac{\partial^{(j, k)}}{\partial \lambda^{(j, k)}} p(\lambda)\right) 2^{-n\left(\alpha_{1}-j\right)} 2^{-m\left(\alpha_{2}-k\right)}\right. \\
& \left.\cdot \delta^{\left(\alpha_{1}-j\right)}\left(2^{-n} \lambda_{1}\right) \delta^{\left(\alpha_{2}-k\right)}\left(2^{-m} \lambda_{2}\right)\right] .
\end{aligned}
$$

Therefore, using (5.5) we obtain that

$$
\begin{aligned}
\left|\frac{\partial^{\alpha}}{\partial \lambda^{\alpha}} p_{n, m}(\lambda)\right| & \leq 2^{-n \alpha_{1}} 2^{-m \alpha_{2}} \sum_{j, k}\left(\begin{array}{c}
\alpha_{1} \\
j
\end{array}\right)\left(\begin{array}{c}
\alpha_{2} \\
k
\end{array}\right) \frac{C_{(j, k)} 2^{n j} 2^{m k}}{\lambda_{1}^{j} \lambda_{2}{ }^{k}} \\
& \leq C(\alpha) 2^{-n \alpha_{1}} 2^{-m \alpha_{2}} \sum_{j, k}\left(\begin{array}{c}
\alpha_{1} \\
j
\end{array}\right)\left(\begin{array}{c}
\alpha_{2} \\
k
\end{array}\right) \frac{2^{n j} 2^{m k} 2^{j+k}}{2^{n j} 2^{m k}} \\
& \leq C(\alpha) 2^{-n \alpha_{1}} 2^{-m \alpha_{2}} \sum_{j, k}\left(\begin{array}{c}
\alpha_{1} \\
j
\end{array}\right)\left(\begin{array}{c}
\alpha_{2} \\
k
\end{array}\right) 2^{j+k} \\
& =3^{\alpha_{1}+\alpha_{2}} C(\alpha) 2^{-n \alpha_{1}} 2^{-m \alpha_{2}}=: \bar{C}_{\alpha} 2^{-n \alpha_{1}} 2^{-m \alpha_{2}},
\end{aligned}
$$

where $C(\alpha)=\max C_{(j, k)}$ and, in going from the first line to the second, we used that $\lambda_{1} \geq 2^{n-1}$ and $\lambda_{2} \geq 2^{m-1}$. Define now $f_{n, m}(\lambda)=p_{n, m}\left(2^{n} \lambda_{1}, 2^{m} \lambda_{2}\right) e^{\lambda_{1}+\lambda_{2}}$. Then

$$
\operatorname{supp} f_{n, m}(\lambda) \subseteq[1 / 2,2] \times[1 / 2,2]
$$


If $\alpha=\left(\alpha_{1}, \alpha_{2}\right) \in \mathbb{N}^{2}$ then using Leibniz's rule one more time we obtain that

$$
\begin{aligned}
\frac{\partial^{\alpha}}{\partial \lambda^{\alpha}} f_{n, m}(\lambda) & =\frac{\partial^{\alpha_{1}}}{\partial \lambda^{\alpha_{1}}} \frac{\partial^{\alpha_{2}}}{\partial \lambda_{2}^{\alpha_{2}}}\left(p_{n, m}\left(2^{n} \lambda_{1}, 2^{m} \lambda_{2}\right) e^{\lambda_{1}+\lambda_{2}}\right) \\
& =\frac{\partial^{\alpha_{1}}}{\partial \lambda^{\alpha_{1}}}\left(\sum_{k=0}^{\alpha_{2}}\left(\begin{array}{c}
\alpha_{2} \\
k
\end{array}\right) 2^{m k}\left(\frac{\partial^{k}}{\partial \lambda_{2}^{k}} p_{n, m}\left(2^{n} \lambda_{1}, 2^{m} \lambda_{2}\right)\right) e^{\lambda_{1}+\lambda_{2}}\right) \\
& =\sum_{j=0}^{\alpha_{1}} \sum_{k=0}^{\alpha_{2}}\left(\begin{array}{c}
\alpha_{1} \\
j
\end{array}\right)\left(\begin{array}{c}
\alpha_{2} \\
k
\end{array}\right) 2^{n j} 2^{m k} \frac{\partial^{(j, k)}}{\partial \lambda^{(j, k)}} p_{n, m}\left(2^{n} \lambda_{1}, 2^{m} \lambda_{2}\right) e^{\lambda_{1}+\lambda_{2}}
\end{aligned}
$$

Thus, using (5.9) and (5.10) we obtain that

$$
\begin{aligned}
\left|\frac{\partial^{\alpha}}{\partial \lambda^{\alpha}} f_{n, m}(\lambda)\right| & \leq \sum_{j=0}^{\alpha_{1}} \sum_{k=0}^{\alpha_{2}}\left(\begin{array}{c}
\alpha_{1} \\
j
\end{array}\right)\left(\begin{array}{c}
\alpha_{2} \\
k
\end{array}\right) 2^{n j} 2^{m k} \bar{C}_{(j, k)} 2^{-n j} 2^{-m k} e^{4} \\
& \leq \bar{C}(\alpha) 2^{\alpha_{1}+\alpha_{2}} e^{4}=: A_{\alpha}
\end{aligned}
$$

Therefore the Fourier transform of $f_{n, m}$ satisfies

$$
\left|\left(\frac{\partial^{\alpha}}{\partial \lambda^{\alpha}} f_{n, m}\right)^{\wedge}(\xi)\right| \leq B_{\alpha}
$$

for all $\alpha \in \mathbb{N}^{2}$, where $B_{\alpha}>0$, and, thus,

$$
\left|\widehat{f}_{n, m}(\xi)\right| \leq \frac{\bar{D}_{\alpha}}{\left(1+\xi_{1}^{2}\right)^{\alpha_{1}}\left(1+\xi_{2}^{2}\right)^{\alpha_{2}}}
$$

for all $\xi=\left(\xi_{1}, \xi_{2}\right) \in \mathbb{R}^{2}$ and $\alpha \in \mathbb{N}^{2}$, where $\bar{D}_{\alpha}$ are positive constants depending only on $\alpha$. Using the inverse Fourier transform we have

$$
p_{n, m}\left(2^{n} \lambda_{1}, 2^{m} \lambda_{2}\right) e^{\lambda_{1}+\lambda_{2}}=\frac{1}{2 \pi} \int \widehat{f}_{n, m}(\xi) e^{i \xi \cdot \lambda} d \xi_{1} d \xi_{2} .
$$

Therefore

$$
p_{n, m}\left(\lambda_{1}, \lambda_{2}\right)=\frac{1}{2 \pi} \int \widehat{f}_{n, m}(\xi) e^{-\lambda_{1}\left(2^{-n}-i \xi_{1} 2^{-n}\right)} e^{-\lambda_{2}\left(2^{-m}-i \xi_{2} 2^{-m}\right)} d \xi_{1} d \xi_{2} .
$$

Thus by spectral theory we have

$$
\begin{aligned}
p_{n, m}( & -\Delta) u_{1}\left(x_{1}\right) u_{2}\left(x_{2}\right) \\
& =\frac{1}{2 \pi} \int \widehat{f}_{n, m}(\xi) e^{\Delta_{1}\left(2^{-n}-i \xi_{1} 2^{-n}\right)} u\left(x_{1}\right) e^{\Delta_{2}\left(2^{-m}-i \xi_{2} 2^{-m}\right)} u\left(x_{2}\right) d \xi .
\end{aligned}
$$

Hence the kernel of $p_{n, m}(-\Delta)$ is

$$
K_{n, m}(x, y)=\frac{1}{2 \pi} \int \widehat{f}_{n, m}(\xi) h_{2^{-n}-i \xi_{1} 2^{-n}}^{(1)}\left(x_{1}, y_{1}\right) h_{2^{-m}-i \xi_{2} 2^{-m}}^{(2)}\left(x_{2}, y_{2}\right) d \xi_{1} d \xi_{2}
$$


for all $x=\left(x_{1}, x_{2}\right), y=\left(y_{1}, y_{2}\right) \in X$, where $h^{(i)}$ is the heat kernel corresponding to $\Delta_{i}, i=1,2$. Using inequalities (3.14) and (5.12) we obtain that

$$
\begin{aligned}
\left|K_{n, m}(x, y)\right| \leq & C(d, j) 2^{\frac{n d_{1}}{d_{1}+1}} \int \exp \left(-\frac{c \gamma R_{1}(x, y)^{\left(d_{1}+1\right) \gamma} 2^{n \gamma_{1}}}{2\left(1+\xi_{1}^{2}\right)^{\frac{\gamma_{1}+1}{2}}}\right) \frac{1}{\left(1+\xi_{1}^{2}\right)^{j}} d \xi_{1} \\
& \cdot 2^{\frac{m d_{2}}{d_{2}+1}} \int \exp \left(-\frac{c \gamma_{2} R_{2}(x, y)^{\left(d_{2}+1\right) \gamma_{2}} 2^{m \gamma_{2}}}{2\left(1+\xi_{2}^{2}\right)^{\frac{\gamma_{2}+1}{2}}}\right) \frac{1}{\left(1+\xi_{2}^{2}\right)^{k}} d \xi_{2},
\end{aligned}
$$

for all $j, k \geq 1$. Lemma (3.5) implies that (if we choose $j$ and $k$ large enough)

$$
\left|K_{p}(x, y)\right|=\left|\sum_{m \in \mathbb{Z}} \sum_{n \in \mathbb{Z}} K_{n, m}(x, y)\right| \leq C(d) R_{1}\left(x_{1}, y_{1}\right)^{-d_{1}} R_{2}\left(x_{2}, y_{2}\right)^{-d_{2}} .
$$

Repeating the above proof for the function $q_{\beta_{1}, \beta_{2}}(\lambda)=\lambda_{i}^{\beta_{1}} \lambda_{j}^{\beta_{2}} p(\lambda)$, we obtain that the kernel $K_{p}(x, y)$ is smooth off the diagonal and satisfies the following estimates:

$$
\left|\Delta_{x, i}^{\beta_{1}} \Delta_{y, j}^{\beta_{2}} K_{p}(x, y)\right| \leq C\left(d, \beta_{1}, \beta_{2}\right) R_{1}(x, y)^{-\beta_{1}\left(d_{1}+1\right)-d_{1}} R_{2}\left(x_{2}, y_{2}\right)^{-\beta_{2}\left(d_{2}+1\right)-d_{2}} .
$$

Corollary 5.5. Assume that $X$ is a product of fractafolds without boundary. If $p \in S^{0}$ then $p(-\Delta)$ is a Calderón-Zygmund operator and, thus, it extends to a bounded operator on $L^{q}(\mu)$ for all $1<q<\infty$ and satisfies weak $1-1$ estimates.

Proof. Theorem 5.4 and Theorem 6.1 of [19] imply that $p(-\Delta)$ is a CalderónZygmund operator.

Remark 5.6. The boundedness of $p(-\Delta)$ on $L^{q}(\mu)$ for all $1<q<\infty$ can be deduced also from the results of [28].

Corollary 5.7. If $p \in S^{m}$ then $p(-\Delta)$ is given by integration with respect to a kernel that is smooth off the diagonal.

Example 5.8. Consider the Riesz potentials (see page 591 of [37])

$$
R_{i} u=\sum \frac{\lambda_{i}}{\lambda_{1}+\cdots+\lambda_{N}} P_{\lambda_{1}} \otimes P_{\lambda_{2}} \otimes \cdots \otimes P_{\lambda_{N}} u, \quad i=1, \ldots, N .
$$

Clearly $p_{i}\left(\lambda_{1}, \ldots, \lambda_{N}\right)=\lambda_{i} /\left(\lambda_{1}+\cdots+\lambda_{N}\right), i=1, \ldots N$, are in $S^{0}$ and thus the Riesz potentials are bounded on $L^{p}(\mu)$, for $1<p<\infty$, and are given by integration with respect to smooth kernels.

\section{Elliptic and hypoelliptic operators}

In this section we specialize the set up of Section 5 to the case when $X$ is the product of $N$ fractafolds without boundary. If $X_{i}$ is not compact, we assume that $\Delta_{i}$ has pure point spectrum. We still write $\lambda=\left(\lambda_{1}, \ldots, \lambda_{N}\right) \in(0, \infty)^{N}, x=$ $\left(x_{1}, \ldots, x_{N}\right) \in X$ and $\Delta=\left(\Delta_{1}, \ldots, \Delta_{N}\right)$. We begin by extending the definition of elliptic operators from Definition 8.2 in [25] to elliptic pseudo-differential operators. 
We show that any pseudo-differential operator satisfies the pseudo-local property and the converse inclusion is also true for elliptic operators. In other words, we prove that elliptic operators on fractals are hypoelliptic. These results give a positive answer to some open questions posed in [36] and [25].

We use the theory of distributions on fractals as developed in [25] for our definitions and results. Most of the results, however, are true for the more general metric spaces that we consider in Section 2 if one replaces the word "distribution" by "function" in what follows.

Recall that the space of test functions $\mathcal{D}(X)$ consists of all smooth functions with compact support. The space of distributions on $X$ is the dual space $\mathcal{D}^{\prime}(X)$ of $\mathcal{D}(X)$ with the weak-star topology (see Definition 4.1 in [25]). A distribution $u$ is smooth on the open set $\Omega \subset X$ (see Definition 8.1 in [25]) if there exists a smooth function $f$ on $\Omega$ such that

$$
\langle u, \phi\rangle=\int f \phi d \mu
$$

for all $\phi \in \mathcal{D}(\Omega)$. If $\Omega_{u}$ is the maximal open set on which $u$ is smooth, then the singular support of $u$ is (see Definition 8.2 in [25])

$$
\operatorname{sing} \operatorname{supp}(u)=X \backslash \Omega_{u} \text {. }
$$

If $p \in S^{m}$ then one can extend the definition of $p(-\Delta)$ to the space of distributions via

$$
\langle p(-\Delta) u, \phi\rangle=\langle u, p(-\Delta) \phi\rangle, \quad \text { for all } \phi \in \mathcal{D}(X)
$$

Definition 6.1. We say that $p(-\Delta)$ satisfies the pseudo-local property if

$$
\operatorname{sing} \operatorname{supp}(p(-\Delta) u) \subseteq \operatorname{sing} \operatorname{supp}(u)
$$

Theorem 6.2. If $p$ is in $S^{m}$ then $p(-\Delta)$ satisfies the pseudo-local property.

Proof. Let $u \in \mathcal{D}^{\prime}(X)$ and let $K_{u}=\operatorname{sing} \operatorname{supp}(u)$. Then $\left.u\right|_{\Omega}$ is smooth, where $\Omega=X \backslash K_{u}$. We need to show that $\left.P u\right|_{\Omega}$ is also smooth. Let $K$ be be any finite union of cells in $\Omega$ and let $\Omega^{\prime}$ the interior of $K$. We identify $\left.u\right|_{\Omega}$ with the smooth functions given by (6.1). Then, using Theorem 7.7 in [25], we can find a smooth function $u_{1}$ such that $\left.u_{1}\right|_{K}=\left.u\right|_{K}$, and $\left.u_{1}\right|_{\Omega^{c}}=0$. We write then $u=u_{1}+u_{2}$, where $u_{2}$ is supported on $K^{c}$. Then $P u=P u_{1}+P u_{2}$. Since $u_{1}$ is smooth it follows that $P u_{1}$ is smooth. Therefore we only need to prove that $\left.P u_{2}\right|_{\Omega^{\prime}}$ is smooth. For this we are going to use the structure theorem for distributions (Theorem 5.10 in [25]; see also the comments following Assumption 7.2 in the same paper). Then $u_{2}$ may be written as a locally finite sum of the form $u_{2}=\sum \Delta^{m_{j}} \nu_{j}$ or $u_{2}=\sum \Delta^{m_{j}+1} f_{j}$, where the $\nu_{j}$ are Radon measures and the $f_{j}$ are continuous functions with compact support. Notice that the support of each $\nu_{j}$ is a subset of $K^{c}$ and, respectively, the support of each $f_{j}$ is a subset of $K^{c}$. 
Assume first that $u_{2}=\sum \Delta^{m_{j}} \nu_{j}$, where the $\nu_{j}$ are Radon measures, and let $\phi \in \mathcal{D}\left(\Omega^{\prime}\right)$. Then

$$
\begin{aligned}
\left\langle p(-\Delta) u_{2}, \phi\right\rangle & =\sum_{j} \int \Delta^{m_{j}} P \phi(x) d \nu_{j}(x)=\sum \int \Delta^{m_{j}} \int K_{p}(x, y) \phi(y) d \mu(y) d \nu_{j}(x) \\
& =\int \sum \int \Delta^{m_{j}} K_{p}(x, y) d \nu_{j}(x) \phi(y) d \mu(y),
\end{aligned}
$$

where $K_{p}$ is the kernel of $p(-\Delta)$ provided by Theorem 5.4. The above expression makes sense because the support of $\phi$ is a subset of $\Omega^{\prime}$, while the supports of $\nu_{j}$ are subsets of $K^{c}$. Since $K_{p}$ is smooth it follows that the function $x \mapsto \sum \int \Delta^{m_{j}} K_{p}(x, y) d \nu_{j}(y)$ is smooth for all $x \in \Omega^{\prime}$. Thus $\left.p(-\Delta) u_{2}\right|_{\Omega^{\prime}}$ is smooth. A similar proof shows that $\left.p(-\Delta) u_{2}\right|_{\Omega^{\prime}}$ is smooth if $u_{2}=\sum \Delta^{m_{j}+1} f_{j}$. It follows that $p(-\Delta) u$ is smooth on $\Omega^{\prime}$. Since $K$ was chosen arbitrarily, we have that $p(-\Delta) u$ is smooth on $\Omega$.

We turn now to the study of elliptic pseudo-differential operators. The first part of the following definition is from Definition 8.4 in [25].

Definition 6.3. An elliptic polynomial of degree $m$ on $\mathbb{R}^{N}$ is a polynomial $q$ of degree $m$ satisfying the property that there exist positive constants $c$ and $A$ such that

$$
|q(\lambda)| \geq c|\lambda|^{\frac{m}{d+1}} \quad \text { for all } \lambda \in \mathbb{R}_{+}^{N} \text { such that }|\lambda| \geq A .
$$

An elliptic differential operator on $X$ is an operator $q(-\Delta)$ for some elliptic polynomial $q(\lambda)$. More generally, an elliptic pseudo-differential operator of order $m$ on $X$ is a pseudo-differential operator $p(-\Delta)$ whose symbol $p \in S^{m}$ satisfies (6.2).

Remark 6.4. Notice that we only require that condition (6.2) holds for nonnegative values of $\lambda_{1}, \ldots, \lambda_{N}$. In particular, the polynomial $p(\lambda)=\lambda_{1}+\cdots+\lambda_{N}$ is elliptic according to our definition.

Theorem 6.5. An elliptic pseudo-differential operator $p(-\Delta)$ is hypoelliptic, meaning that

$$
\operatorname{sing} \operatorname{supp}(p(-\Delta) u)=\operatorname{sing} \operatorname{supp}(u) .
$$

Proof. Since $p$ is elliptic the set of its zeros is compact and $p(\lambda) \neq 0$ for all $\lambda \in \mathbb{R}^{N}$ with $|\lambda| \geq A$. Let $\xi \in C^{\infty}$ be a cut-off function which is 0 on a neighborhood of the zeros of $p$ and is 1 for $|\lambda| \geq A$. Define $p_{1}(\lambda)=\xi(\lambda) / p(\lambda)$, so that $p_{1}(\lambda)$ is zero on a neighborhood of the zeros of $p$. Then $p(\lambda) p_{1}(\lambda)=1$ if $|\lambda| \geq A$. Moreover $p_{1} \in S^{-m}$, since for $|\lambda| \geq A$ we have that (see, for example, the proof of Theorem 1.3 in [41])

$$
\frac{\partial^{\alpha}}{\partial \lambda^{\alpha}} p_{1}(\lambda)=\sum_{\alpha^{1}+\alpha^{2}+\cdots+\alpha^{\mu}=\alpha} \frac{\partial^{\alpha^{1}}}{\partial \lambda^{\alpha^{1}}} p(\lambda) \cdots \frac{\partial^{\alpha^{\mu}}}{\partial \lambda^{\alpha^{\mu}}} p(\lambda) \cdot p(\lambda)^{1-\mu} .
$$

Using Lemma 3.4 and Theorem 5.4 we have that

$$
p_{1}(-\Delta) p(-\Delta) u=u+R u,
$$

where $R \in S^{-\infty}$ has a smooth compactly supported symbol $r(\lambda)$. Hence $R$ is infinitely smoothing. It follows that if $\Omega$ is an open set such that $\left.p(-\Delta) u\right|_{\Omega}$ is 
smooth, then $\left.u\right|_{\Omega}=\left.p_{1}(-\Delta) p(-\Delta) u\right|_{\Omega}-\left.R u\right|_{\Omega}$ is smooth. Therefore sing $\operatorname{supp}(u) \subseteq$ $\operatorname{sing} \operatorname{supp}(p(-\Delta) u)$. Since the converse is provided by Theorem 6.2 , it follows that $p(-\Delta)$ is hypoelliptic.

\subsection{Quasi-elliptic operators}

It is known $([6],[10])$ that for some fractals the set of ratios of eigenvalues of the Laplacian has gaps. That is, there are $\alpha<\beta$ such that $\lambda / \lambda^{\prime} \notin(\alpha, \beta)$ for all $\lambda \neq \lambda^{\prime}$ in the spectrum of $-\Delta$. Consider the product $X=X_{1} \times X_{2}$ of two copies of the same fractafold without boundary that has a spectral gap $(\alpha, \beta)$. Let $\Delta_{1}$ and $\Delta_{2}$ be the Laplacian on the fractafold viewed as acting on $X_{1}$ and, respectively, on $X_{2}$. As before, we write $\Delta=\left(\Delta_{1}, \Delta_{2}\right)$. For a real number $a$ in $(\alpha, \beta)$ and $\varepsilon>0$ define the cone

$$
\Gamma_{a, \varepsilon}=\left\{\left(\lambda_{1}, \lambda_{2}\right) \in \mathbb{R}_{+}^{2}:\left|\lambda_{1}-a \lambda_{2}\right|<\varepsilon \lambda_{2}\right\} .
$$

If $a \in(\alpha, \beta)$ then there is $\varepsilon>0$ such that $\Gamma_{a, \varepsilon}$ does not contain any pair of eigenvalues of $-\Delta$. Let $\mathfrak{G}$ be the collection of such cones. Denote by $\mathcal{G}$ the collection of cones in $\mathbb{R}_{+}^{2}$ that do not intersect any cone in $\mathfrak{G}$. Thus any pair of eigenvalues of $-\Delta$ lies in some cone in $\mathcal{G}$. Moreover, any pseudo-differential operator will "live" on $\mathcal{G}$, as we will make precise in Lemma 6.9.

Definition 6.6. We say that an $m$-symbol $p$ is quasi-elliptic if it satisfies (6.2) for all $\lambda \in \Gamma$ and $\Gamma \in \mathcal{G}$, with the constant $A$ depending on $\Gamma$. A pseudo-differential operator $p(-\Delta)$ is called a quasi-elliptic operator if $p$ is a quasi-elliptic symbol.

Example 6.7. If $a \in(\alpha, \beta)$ then the operator $\Delta_{1}-a \Delta_{2}$ is a quasi-elliptic operator ([6], [28]). An intriguing consequence of the existence of spectral gaps is that, if $a \in(\alpha, \beta)$, then the operator $1 /\left(\Delta_{1}-a \Delta_{2}\right)$ is bounded on $L^{p}(\mu)$ for all $1<p<\infty$ (see $[28])$. The next proposition show that the quasi-elliptic operators are, in fact, elliptic operators.

Proposition 6.8. A quasi-elliptic pseudo-differential operator $p(-\Delta)$ is equal to an elliptic pseudo-differential operator.

This proposition follows immediately from the following lemma which says that a quasi-elliptic symbol equals an elliptic symbol outside a cone contained in the spectral gaps. Note that even if $p(-\Delta)$ is a differential operator, the elliptic operator is only a pseudo-differential operator.

Lemma 6.9. Let $p$ be a quasi-elliptic m-symbol. Then there is an elliptic $m$-symbol $\tilde{p}$ such that $p(\lambda)=\tilde{p}(\lambda)$ for all $\lambda \in \Gamma$ and all $\Gamma \in \mathcal{G}$.

Proof. Let $a \in(\alpha, \beta)$ and $\varepsilon>0$ be such that the cone $\Gamma_{a, \varepsilon}$ is a maximal cone in $\mathfrak{G}$ and let $\Gamma=\mathbb{R}_{+}^{2} \backslash \Gamma_{a, \varepsilon}$. Then $\Gamma$ is a union of two simply connected cones. Since $p$ is quasi-elliptic, there are $c>0$ and $A>0$ such that $|p(\lambda)| \geq c|\lambda|$ for all $\lambda \in \Gamma$ with $|\lambda|>A$. Since $\Gamma_{A}:=\{\lambda \in \Gamma:|\lambda|>A\}$ is a union of two disjoint simply connected sets, we can define the function $q(\lambda)=\log p(\lambda)$. Let $r(\lambda)$ and $s(\lambda)$ be the real part and the imaginary part, respectively, of $q(\lambda)$. By hypothesis, $r(\lambda) \geq \frac{m}{d+1} \log |\lambda|$ for 
all $\lambda \in \Gamma_{A}$. Let $\tilde{r}(\lambda)$ and $\tilde{s}(\lambda)$ be two smooth extensions of $r(\lambda)$ and $s(\lambda)$ such that $\tilde{r}(\lambda) \geq \frac{m}{d+1} \log |\lambda|$ for all $\lambda \in \mathbb{R}_{+}^{2}$ with $|\lambda|>A$. Let $\tilde{p}(\lambda)=\exp (\tilde{r}(\lambda)+i \tilde{s}(\lambda))$ and extend it so that $\tilde{p}(\lambda)=p(\lambda)$ for all $\lambda \in \Gamma$. Then $\tilde{p}$ is the desired elliptic $m$-symbol.

Proof of Proposition 6.8. Let $q$ be an elliptic symbol $q$ such that $p(\lambda)=q(\lambda)$ for all $\lambda \in \Gamma$ and all $\Gamma \in \mathcal{G}$. Then $q(-\Delta)$ is an elliptic pseudo-differential operator. Moreover, since $p(\lambda)=q(\lambda)$ for all $\lambda \in \Lambda_{1} \times \Lambda_{2}$ (where $\Lambda_{i}$ is the spectrum of $-\Delta_{i}$ ), it follows from the definition that $p(-\Delta)=q(-\Delta)$. Therefore $p(-\Delta)$ is equal to an elliptic pseudo-differential operator.

As an immediate consequence of the above proposition and Theorem 6.5 we obtain the following corollary, which answers an open question posed in [25].

Corollary 6.10. A quasi-elliptic operator is hypoelliptic.

\section{7. $\rho$-type symbols and pseudo-differential operators}

In this section we define and study pseudo-differential operators for which the derivatives of the symbols have lower rates of decay. These operators still have kernels that are smooth off the diagonal, even though they might not be bounded on $L^{q}$-spaces, as well-known examples in the Euclidean setting show. As an application, we study the so called Hörmander type hypoelliptic operators and prove that they are hypoelliptic in this more general setting. In the following we use the set up of Section 3. The definitions and main theorem of this section can be extended without difficulty to the product setting so we omit the details (see Remark 7.5). In Subsection 7.1 we will use the fact that a result equivalent to Theorem 7.3 holds for products of measure metric spaces.

Definition 7.1. For fixed $m \in \mathbb{R}$ and $0 \leq \rho \leq 1$ define the symbol class $S_{\rho}^{m}$ to be the set of $p \in C^{\infty}((0, \infty))$ with the property that for any $k \geq 0$ there is $C_{k}(\rho)>0$ such that

$$
\left|\left(\lambda^{\rho} \frac{d}{d \lambda}\right)^{k} p(\lambda)\right| \leq C_{k}(\rho)(1+\lambda)^{\frac{m}{d+1}} \quad \text { for all } \lambda>0 .
$$

Definition 7.2. Define the operator class $\Psi D O_{\rho}^{m}$ by

$$
p(-\Delta) u=\int_{0}^{\infty} p(\lambda) P(\lambda) u \quad \text { for } p \in S_{\rho}^{m} \text { and } u \in \mathcal{D} .
$$

Theorem 7.3. Let $\frac{1}{\gamma+1}<\rho \leq 1$ and let $p:(0, \infty) \rightarrow \mathbb{C}$ be an $S_{\rho}^{0}$-symbol, that is, for each $k \geq 0$ there is $C_{k}(\rho)>0$ such that

$$
\left|\lambda^{\rho k} \frac{\partial^{k}}{\partial \lambda^{k}} p(\lambda)\right| \leq C_{k}(\rho) .
$$

Then $p(-\Delta)$ has a kernel $K(x, y)$ that is smooth off the diagonal of $X \times X$.

Proof. The proof of this theorem follows the lines of the proof of Theorem 3.6. We will present the main steps of the proof skipping the computations, as they are very 
similar. Let $\delta$ be the function from the Littlewood-Paley decomposition and let $p_{n}(\lambda)=p(\lambda) \delta\left(2^{-n} \lambda\right)$. Then $\operatorname{supp} p_{n} \subseteq\left[2^{n-1}, 2^{n+1}\right]$ and

$$
\left|\frac{d^{k}}{d \lambda^{k}} p_{n}(\lambda)\right| \lesssim 2^{-n \rho k} \quad \text { for all } k \geq 0 .
$$

Define $\tilde{p}_{n}(\lambda)=p_{n}\left(2^{n \rho} \lambda\right)$. Then $\operatorname{supp} \widetilde{p}_{n} \subseteq\left[2^{n(1-\rho)-1}, 2^{n(1-\rho)+1}\right]$ and for each $k \geq 0$ there is a constant $C_{k}(\rho)>0$ such that

$$
\left|\frac{d^{k} \widetilde{p}_{n}(\lambda)}{d \lambda^{k}}\right| \leq C_{k}(\gamma)
$$

Let $f_{n}(\lambda)=\widetilde{p}_{n}(\lambda) e^{\frac{\lambda}{2^{n(1-\rho)}}}$. Since $e^{\frac{\lambda}{2^{n(1-\rho)}}} \leq e^{2}$ for all $\lambda \in\left[2^{n(1-\rho)-1}, 2^{n(1-\rho)+1}\right]$, it follows that for each $k \geq 0$ there is a constant $A_{k}(\gamma)>0$ independent of $n$ such that

$$
\left|\frac{d^{k} f_{n}(\lambda)}{d \lambda^{k}}\right| \leq A_{k}(\gamma)
$$

Therefore

$$
\left|\xi^{k} \widehat{f}_{n}(\xi)\right| \lesssim 1 \quad \text { for all } k \geq 0
$$

In particular we have that for each $k \geq 0$ there is a constant $\bar{D}_{k}(\gamma)$ independent of $n$ such that

$$
\left|\widehat{f}_{n}\left(2^{n(\rho-1)} \xi\right)\right| \leq \frac{\bar{D}_{k}(\gamma) 2^{2 n k(1-\rho)}}{\left(1+\xi^{2}\right)^{k}} \text { for all } k \geq 0 .
$$

Using the Fourier inversion formula we have that

$$
p_{n}\left(2^{n \rho} \lambda\right) e^{\frac{2^{n \rho} 2_{\lambda}}{2^{n}}}=\frac{1}{2 \pi} \int \widehat{f}_{n}(\xi) e^{i \lambda \xi} d \xi
$$

Therefore

$$
p_{n}(\lambda)=\frac{1}{2 \pi} \int \widehat{f}_{n}(\xi) e^{-\lambda\left(2^{-n}-i \xi 2^{-n \rho}\right)} d \xi=\frac{1}{2 \pi} \int \widehat{f}_{n}(\xi) e^{-\lambda 2^{-n}\left(1-i \xi 2^{-n(\rho-1)}\right)} d \xi,
$$

which, using the substitution $u=\xi 2^{-n(\rho-1)}$, becomes

$$
p_{n}(\lambda)=\frac{2^{n(\rho-1)}}{2 \pi} \int \widehat{f}_{n}\left(2^{n(1-\rho)} \xi\right) e^{-\lambda 2^{-n}(1-i \xi)} d \xi
$$

Thus the kernel of $p_{n}(-\Delta)$ equals

$$
K_{n}(x, y)=\frac{2^{n(\rho-1)}}{2 \pi} \int \widehat{f}_{n}\left(2^{n(1-\rho)} \xi\right) h_{2^{-n}-i \xi 2^{-n}}(x, y) d \xi
$$

Using the estimates (3.14) and (7.2) we obtain that

$$
\begin{aligned}
\left|K_{n}(x, y)\right| \lesssim & 2^{\frac{n}{d+1}(d+(1-\rho)(d+1)(2 j-1))} \\
& \cdot \int \exp \left(-\frac{c \gamma}{2} R(x, y)^{(d+1) \gamma} 2^{n \gamma}\left(1+\xi^{2}\right)^{-\frac{\gamma+1}{2}}\right) \frac{1}{\left(1+\xi^{2}\right)^{j}} d \xi
\end{aligned}
$$


for all $j \geq 0$. Lemma 3.5 implies that if we pick $j$ such that

$$
j \geq \frac{(d+(1-\rho)(d+1)(2 j-1)(\gamma+1)}{2 \gamma(d+1)}+\frac{1}{2}
$$

then $K(x, y)=\sum_{n} K_{n}(x, y)$ converges. We can pick a positive $j$ in the equation above provided that $\rho>1 /(\gamma+1)$. Then it follows that the kernel $K$ satisfies the estimates

$$
|K(x, y)| \lesssim R(x, y)^{-\frac{d \gamma}{\rho(\gamma+1)-1}}
$$

A similar argument for $q(\lambda)=\lambda^{j} p(\lambda), j \geq 1$, shows that $K$ is smooth off the diagonal.

Corollary 7.4. If $1 /(\gamma+1)<\rho \leq 1$ and $p \in S_{\rho}^{m}$ then the kernel of $p(-\Delta)$ is smooth off the diagonal.

Proof. The proof is very similar to that of Corollary 3.9.

Remark 7.5. Note that for $\rho<1$ the operators $p(-\Delta)$ are not Calderón-Zygmund operators and they might not extend to $L^{q}$ for all values of $q$. Also, one can easily extend the definition to product of fractals and adapt the proof of Theorem 5.4 to show that the kernel of a pseudo-differential operator of type $\rho$ with $1 /(\gamma+1)<$ $\rho \leq 1$ is smooth off the diagonal.

\subsection{Hörmander type hypoelliptic operators}

Let $X$ be a product of $N$ measure metric space as in Section 5. Recall that we write $\Delta=\left(\Delta_{1}, \ldots, \Delta_{N}\right)$.

Definition 7.6. ([17]) We say that a smooth map $p:(0, \infty)^{N} \rightarrow \mathbb{C}$ is a Hörmander type hypoelliptic symbol if there are $\varepsilon>0$ and $A>0$ such that

$$
\left|\frac{\frac{\partial^{\alpha}}{\partial \lambda^{\alpha}} p(\lambda)}{p(\lambda)}\right| \leq c_{\alpha}|\lambda|^{-\varepsilon|\alpha|} \quad \text { for }|\lambda| \geq A,
$$

where $c_{\alpha}$ are positive constants for all $\alpha \in \mathbb{N}^{N}$.

Theorem 7.7. Suppose that $p$ is a Hörmander type hypoelliptic symbol with $\varepsilon>$ $1 /(\gamma+1)$. Then $p(-\Delta)$ is hypoelliptic.

Proof. We know from (7.3) that the set of zeros of $p$ is compact. Let $\varphi$ be a smooth function that is 0 on a neighborhood of the zeros of $p$ and $\varphi(\lambda)=1$ if $|\lambda| \geq A$. Set $q(\lambda)=\varphi(\lambda) p(\lambda)^{-1}$. Then, if $|\lambda| \geq A$,

$$
\frac{\partial^{\alpha}}{\partial \lambda^{\alpha}} q(\lambda)=\sum_{\alpha_{1}+\cdots+\alpha_{\mu}=\alpha} \frac{\partial^{\alpha_{1}}}{\partial \lambda^{\alpha_{1}}} p(\lambda) \cdots \frac{\partial^{\alpha_{\mu}}}{\partial \lambda^{\alpha_{\mu}}} p(\lambda) \cdot p(\lambda)^{1-\mu},
$$

It follows that $q \in S_{\varepsilon}^{0}$. Since $q(\lambda) p(\lambda)=1$ if $|\lambda| \geq A$ we have that

$$
q(-\Delta) p(-\Delta)=u+R u,
$$

where $R$ is an infinitely smoothing operator. Theorem 7.3 implies that $p(-\Delta)$ is hypoelliptic. 


\section{Wavefront set and microlocal analysis}

In this section we initiate the study of microlocal analysis on fractals. Namely, we define the wavefront sets on fractals and provide a few concrete examples. As in classical harmonic analysis, the wavefront sets contain information about the singularities of distributions. We show that, in general, a pseudo-differential operator reduces the wavefront set. If the operator is elliptic, then the wavefront set remains unchanged. This is just a beginning of this study. We expect that the wavefront set will play an important role in the future, but we cannot expect analogues of classical results showing that the wavefront set controls propagation of singularities for space-time equations, as there are localized eigenfunctions which prevent singularities from propagating at all. We continue to assume the set up and notations of Section 6. Namely, $X$ is the product of $N$ fractafolds without boundary, $\mu$ is the product measure on $X$, and $\Delta=\left(\Delta_{1}, \ldots, \Delta_{N}\right)$. In addition, we assume in this section that each fractafold $X_{i}$ is compact.

Definition 8.1. Let $\Gamma$ denote an open cone in $\mathbb{R}_{+}^{N}$ and $\Omega$ an open set in $X$. We use $\varphi_{\alpha_{k}}$ to denote $L^{2}$ normalized eigenfunctions corresponding to eigenvalues $\lambda_{\alpha_{k}}$, and set $\lambda_{\alpha}=\lambda_{\alpha_{1}}+\cdots+\lambda_{\alpha_{N}}$. A distribution $u$ is defined to be $C^{\infty}$ in $\Omega \times \Gamma$ if it can be written on $\Omega$ as a linear combination of eigenfunctions with coefficients having faster than polynomial decay over the eigenvalues in $\Gamma$. More precisely, there is a sequence $b_{n}$ and a function $v$ with $\left.v\right|_{\Omega}=u$ that has the form

$$
v=\sum_{\alpha} c_{\alpha} \varphi_{\alpha_{1}} \otimes \varphi_{\alpha_{2}} \otimes \cdots \otimes \varphi_{\alpha_{N}}
$$

for values $c_{\alpha}$ such that $\left|c_{\alpha}\right| \leq b_{n}\left(1+\lambda_{\alpha}\right)^{-n /(d+1)}$ for all $n$ and all $\left\{\lambda_{\alpha_{1}}, \ldots, \lambda_{\alpha_{N}}\right\} \in \Gamma$. We define the wavefront set of $u, \mathrm{WF}(u)$, to be the complement of the union of the sets where $u$ is $C^{\infty}$.

Remark 8.2. If $u$ is a smooth function on $X$ then $\mathrm{WF}(u)$ is empty. More generally, sing $\operatorname{supp} u$ is the projection of $\operatorname{WF}(u)$ onto $X$.

Remark 8.3. If the fractals $X_{i}$ have gaps in the set of ratios of eigenvalues, then there are special cones $\Gamma$ for which every $u$ is $C^{\infty}$ on $X \times \Gamma$ because $\Gamma$ contains no eigenvalues (see Subsection 6.1).

Proposition 8.4. If $p \in S^{m}$ then $\mathrm{WF}(p(-\Delta) u) \subseteq \mathrm{WF}(u)$. If in addition $p(-\Delta)$ is elliptic then $\mathrm{WF}(p(-\Delta) u)=\mathrm{WF}(u)$.

Proof. Let $u$ be a distribution on $X$ and suppose that $\Omega$ is an open subset of $X$ and $\Gamma$ is an open cone in $\mathbb{R}_{+}^{N}$ such that $u$ is $C^{\infty}$ in $\Omega \times \Gamma$. Let $v$ be as in (8.1). Then $\left.P v\right|_{\Omega}=P u$ and

$$
P v=\sum_{\alpha} c_{\alpha} p\left(\lambda_{\alpha_{1}}, \ldots, \lambda_{\alpha_{N}}\right) \varphi_{\alpha_{1}} \otimes \cdots \otimes \varphi_{\alpha_{N}}
$$

with $\left|c_{\alpha} p\left(\lambda_{\alpha_{1}}, \ldots, \lambda_{\alpha_{N}}\right)\right| \leq b_{n}\left(1+\lambda_{\alpha}\right)^{-(n-m) /(d+1)}$ for all $\left\{\lambda_{\alpha_{1}}, \ldots, \lambda_{\alpha_{N}}\right\} \in \Gamma$. Thus $P u$ is $C^{\infty}$ in $\Omega \times \Gamma$ and, since $\Omega$ and $\Gamma$ were arbitrary, $\operatorname{WF}(P u) \subseteq \operatorname{WF}(u)$. 
If $P$ is elliptic then we can write, as in the proof of Theorem $6.5, u=P_{1} P u+R u$, where $P_{1}$ is a pseudo-differential operator of order $-m$ and $R$ is a smoothing operator. The conclusion follows immediately from this.

Example 8.5. Let $N=2$ and let $u=u_{1} \otimes u_{2}$ be a tensor product of distributions. From the definition $u$ is $C^{\infty}$ on $\left(\left(\operatorname{sing} \operatorname{supp} u_{1}\right)^{c} \times\left(\operatorname{sing} \operatorname{supp} u_{2}\right)^{c}\right) \times \mathbb{R}_{2}^{+}$, thus $\mathrm{WF}(u)$ is a subset of the complement of this set. It is also easy to check that

$$
\left(\operatorname{sing} \operatorname{supp}\left(u_{1}\right) \times \operatorname{sing} \operatorname{supp}\left(u_{2}\right)\right) \times \mathbb{R}_{2}^{+} \subseteq \mathrm{WF}(u) .
$$

Let $x_{1} \in X_{1}$ and $x_{2} \in X_{2}$ and suppose that $u_{1}$ is smooth near $x_{1}$ but $x_{2} \in$ $\operatorname{sing} \operatorname{supp}\left(u_{2}\right)$. Then there is a smooth function $v_{1}$ on $X_{1}$ and a neighborhood $\Omega_{1}$ of $x_{1}$ such that $u_{1}=v_{1}$ on $\Omega_{1}$. Moreover we can write $v_{1}=\sum_{j} c_{j} \varphi_{j}$ with the Fourier coefficients $c_{j}$ decaying faster than any polynomial (Theorem 3.5 in [25]). Therefore for any $n \in \mathbb{N}$ there is $b_{n, j}>0$ such that

$$
\left|c_{j}\right| \leq b_{n, j}\left(1+\lambda_{j}\right)^{-n} \text {. }
$$

Also $u_{2}=\sum_{k} c_{k}^{\prime} \varphi_{k}$, where the coefficients $c_{k}^{\prime}=\left\langle u_{2}, \varphi_{k}\right\rangle$ have at most polynomial growth (Lemma 4.4 in [25]). Thus for every $k \in \mathbb{N}$ there are $m_{k} \in \mathbb{N}$ and $b_{k}^{\prime}>0$ such that

$$
\left|c_{k}^{\prime}\right| \leq b_{k}^{\prime}\left(1+\lambda_{k}\right)^{m_{k}}
$$

Define

$$
v=v_{1} \otimes u_{2}=\sum c_{j} c_{k}^{\prime} \varphi_{j} \otimes \varphi_{k}
$$

and let $\Gamma$ be a cone in $\mathbb{R}_{+}^{2} \backslash\{y$-axis $\}$. Then there is $M>0$ such that for all $\left(\lambda^{\prime}, \lambda^{\prime \prime}\right) \in \Gamma$ we have that $\left|\lambda^{\prime \prime}\right| \leq M\left|\lambda^{\prime}\right|$. Therefore

$$
\begin{aligned}
\left|c_{j} c_{k}^{\prime}\right| & \leq b_{n, j}\left(1+\lambda_{j}\right)^{-n} b_{k}^{\prime}\left(1+\lambda_{k}\right)^{m_{k}} \\
& \leq M^{m_{k}} b_{n, j} b_{k}^{\prime}\left(1+\lambda_{j}\right)^{-n+m_{k}} \leq C(M, j, k)\left(1+\lambda_{j}+\lambda_{k}\right)^{-n+m_{k}},
\end{aligned}
$$

and thus $u$ is $C^{\infty}$ on $\left(\Omega_{1} \times X_{2}\right) \times \Gamma$.

Similarly, one can show that if $x_{1} \in \operatorname{sing} \operatorname{supp} u_{1}$ and $u_{2}$ is smooth near $x_{2}$, then $u$ is $C^{\infty}$ on $\left(X_{1} \times\left(\operatorname{sing} \operatorname{supp}\left(u_{2}\right)\right)^{c}\right) \times \Gamma$ for all cones $\Gamma$ in $\mathbb{R}_{+}^{2} \backslash\{x-$ axis $\}$. Therefore,

$$
\begin{aligned}
\mathrm{WF}(u)= & \left(\operatorname{sing} \operatorname{supp}\left(u_{1}\right) \times \operatorname{sing} \operatorname{supp}\left(u_{2}\right)\right) \times \mathbb{R}_{+}^{2} \\
& \bigcup\left(\left(\operatorname{sing} \operatorname{supp}\left(u_{1}\right)\right)^{c} \times \operatorname{sing} \operatorname{supp}\left(u_{2}\right)\right) \times\{y-\text { axis }\} \\
& \bigcup\left(\operatorname{sing} \operatorname{supp}\left(u_{1}\right) \times\left(\operatorname{sing} \operatorname{supp}\left(u_{2}\right)\right)^{c}\right) \times\{x-\text { axis }\} .
\end{aligned}
$$

Example 8.6. We continue to assume that $N=2$. Let $x=\left(x_{1}, x_{2}\right) \in X$ and let $\{(j, k)\}$ be a sequence such that the maps $\varphi_{j} \otimes \varphi_{k}$ are supported in a decreasing sequence of neighborhoods of $x$. Let $\left\{c_{j k}\right\}$ be any sequence of real numbers and define $u=\sum c_{j k} \varphi_{j} \otimes \varphi_{k}$. Let $\Omega$ be an open subset of $X$ such that there is an open neighborhood $V$ of $x$ so that $\Omega \bigcap V=\emptyset$. Then there are only a finite number of indices $(j, k)$ with the property that $\operatorname{supp} \varphi_{j} \otimes \varphi_{k} \bigcap \Omega \neq \emptyset$. It follows that $u$ is $C^{\infty}$ on $\Omega \times \mathbb{R}_{+}^{2}$. Since $\Omega$ was arbitrary, it follows that $\operatorname{WF}(u) \subseteq\{x\} \times \mathbb{R}_{+}^{2}$. Let $\Gamma$ be a cone in $\mathbb{R}_{+}^{2}$. Define $c_{j k}=1$ if $\left(\lambda_{j}, \lambda_{k}\right) \in \Gamma$ and set $c_{j k}$ to be zero otherwise. Then for $u=\sum c_{j k} \varphi_{j} \otimes \varphi_{k}$ we have that $\mathrm{WF}(u)=\{x\} \times \Gamma$. 
More generally, let $K$ be any compact subset of $X^{2}$. Let $\{(j, k)\}$ be a sequence such that $\varphi_{j} \otimes \varphi_{k}$ is supported in a decreasing sequence of neighborhoods of $K$. Then if $u=\sum c_{j k} \varphi_{j} \otimes \varphi_{k}$, with $\left\{c_{j k}\right\}$ an arbitrary sequence, we have that $\mathrm{WF}(u) \subseteq K \times \mathbb{R}_{+}^{2}$. If $\Gamma$ is a cone in $\mathbb{R}_{+}^{2}$ and we define $c_{j k}$ as before, we obtain that $\mathrm{WF}(u)=K \times \mathbb{R}_{+}^{2}$.

\section{Pseudo-differential operators with variable coefficients}

The symbols of the operators studied in the previous section are independent of the $x$ variable. These symbols are also known as constant coefficient symbols, and the corresponding operators are the constant coefficient operators. In this section we extend our study to operators whose symbols depend on both the $\lambda$ and $x$ variables. We call them pseudo-differential operators with variable coefficients. The main difficulty in studying these operators is the fact that for a large class of fractals the domain of the Laplacian is not closed under multiplication [5]. This implies in our case that the symbolic calculus is not valid for the pseudo-differential operators with variable coefficients. Namely, the product of two symbols in the sense of Definition 9.1 is no longer smooth and it cannot be a symbol of a pseudodifferential operator. Another consequence of this fact is that the kernels of these operators cannot be smooth. Nevertheless, we conjecture that these are CalderónZygmund operators in the sense of [31], [19]. We prove, in fact, that the kernels of these operators are continuous off the diagonal and decay correctly off the diagonal. Moreover, we show that these operators are bounded on $L^{q}(\mu)$ for all $1<q<\infty$.

We can define the operators if $X$ is either a compact fractafold without boundary based on a self-similar nested fractal $K$, or an infinite blow-up of $K$ without boundary. However we prove the main properties in the case when $X$ is compact. We continue to write $\mathcal{D}$ for the set of finite linear combinations of eigenvalues of $-\Delta$ with compact support and $\Lambda$ for the spectrum of $-\Delta$. We also assume that the heat kernel satisfies the upper estimate (2.2).

Definition 9.1. For $m \in \mathbb{R}$ we define the symbol class $S^{m}$ to consist of the smooth functions $p: X \times(0, \infty) \rightarrow \mathbb{C}$ such that for each $k \in \mathbb{N}$ there is a positive constant $C_{j, k}$ such that

$$
\left|\left(\lambda \frac{\partial}{\partial \lambda}\right)^{k} \Delta_{x}^{j} p(x, \lambda)\right| \leq C_{j, k}(1+\lambda)^{\frac{m}{d+1}} .
$$

For $\lambda \in \Lambda$ we set $P_{\lambda}$ to be the corresponding spectral projection.

Definition 9.2. We define the operator class of pseudo-differential operators with variable coefficients $\Psi D O_{m}$ by

$$
p(x,-\Delta) u(x)=\sum_{\lambda \in \Lambda} \int p(x, \lambda) P_{\lambda}(x, y) u(y) d y \quad \text { for } p \in S^{m} \text { and } u \in \mathcal{D} .
$$

In the remainder of this section we will assume that $X$ is a compact fractafold without boundary. Recall that the set of eigenvalues is countable and the only 
accumulation point is $\infty$. Let $\Lambda=\left\{\lambda_{1} \leq \lambda_{2} \leq \cdots\right\}$ be the set of eigenvalues of $-\Delta$ in nondecreasing order (repeated according to multiplicity). Let $\left\{\phi_{k}\right\}_{k \in \mathbb{N}}$ be an orthonormal basis of $L^{2}(\mu)$ consisting of compactly supported eigenfunctions.

Theorem 9.3. Suppose that $X$ is a compact fractafold with no boundary and $p \in S^{0}$. Then the operator $T u(x)=p(x,-\Delta) u(x)$ extends to a bounded operator on $L^{2}(\mu)$.

Proof. Define

$$
\widetilde{T} u(x, z)=\sum_{\lambda \in \Lambda} \int p(z, \lambda) P_{\lambda}(x, y) u(y) d y
$$

Then

$$
|T u(x)|=|\widetilde{T} u(x, x)| \leq \sup _{z}|\widetilde{T} u(z, x)| \leq\left\|\left(I-\Delta_{z}\right)^{\frac{d}{2(d+1)}} \widetilde{T} u(\cdot, x)\right\|_{2},
$$

where the last inequality is the Sobolev inequality. So $\|T u\|_{2}^{2} \leq\left\|\left(I-\Delta_{z}\right) \widetilde{T} u\right\|_{2}^{2}$. If $p \in S^{0}$ then $\Delta_{z} p \in L^{\infty}$ because $X$ is compact. Then the result follows.

Remark 9.4. The method of proof for the above theorem is adapted from [33].

Let $K(x, y)$ be the distributional kernel of $T$. Recall that we cannot expect that $K$ is smooth off the diagonal, because the domain of the Laplacian is not, in general, closed under multiplication. The best result one can expect is that $K$ is continuous off the diagonal. We prove that this is true in the following theorem.

Theorem 9.5. Suppose that $X$ is a compact fractafold with no boundary and $p \in S^{0}$. Then the operator $T u(x)=p(x,-\Delta) u(x)$ is given by integration against a kernel that is continuous off the diagonal and satisfies the estimate

$$
|K(x, y)| \lesssim R(x, y)^{-d} .
$$

Proof. Since the map $x \mapsto p(x, \lambda)$ is smooth for all $\lambda \in \mathbb{R}_{+}$, it follows that we can write

$$
p(x, \lambda)=\sum_{k=0}^{\infty} m_{k}(\lambda) \phi_{k}(x),
$$

where $m_{k}(\lambda)=\left\langle p(\cdot, \lambda), \phi_{k}\right\rangle$, for all $\lambda \in \mathbb{R}_{+}$. We claim that $\widetilde{m}_{k, n}(\lambda):=\lambda_{k}^{n} m_{k}(\lambda)$ is a 0 -symbol with constant coefficients, for all $k, n \in \mathbb{N}$. For $j \geq 0$ we have

$$
\begin{aligned}
& \lambda^{j} \frac{d^{j} \tilde{m}_{k, n}(\lambda)}{d \lambda^{j}}=\int \lambda^{j} \frac{\partial^{j}}{\partial \lambda^{j}} p(x, \lambda) \lambda_{k}^{n} \phi_{k}(x) d \mu(x) \\
& \quad=\int \lambda^{j} \frac{\partial^{j}}{\partial \lambda^{j}} p(x, \lambda)(-\Delta)^{n} \phi_{k}(x) d \mu(x)=\int \lambda^{j}(-\Delta)^{n} \frac{\partial^{j}}{\partial \lambda^{j}} p(x, \lambda) \phi_{k}(x) d \mu(x),
\end{aligned}
$$

using the Gauss-Green formula. The estimates (9.1) imply that

$$
\left|\lambda^{j} \frac{d^{j} \widetilde{m}_{k, n}(\lambda)}{d \lambda^{j}}\right| \leq C_{j, n} \int\left|\phi_{k}(x)\right| d \mu(x) \leq C_{j, n} \mu(X)^{1 / 2} .
$$

Thus $\tilde{m}_{k, n}(\lambda)$ is a 0 -symbol. Notice that the constants in (9.4) are independent of $k$. Now Theorem 4.5.4 of [21] (and the comments following it) implies that there 
is a positive constant $c$ and a real number $\alpha$ such that

$$
\left\|\phi_{k}\right\|_{\infty} \leq c \lambda_{k}^{\alpha} \text { for all } k \geq 0
$$

Let $n \geq 0$ be such that the series $S:=\sum_{k} \lambda_{k}^{\alpha-n}$ converges. For $u \in \mathcal{D}$ we can write then

$$
\begin{aligned}
T u(x) & =\sum_{j} \sum_{k} m_{k}\left(\lambda_{j}\right) \phi_{k}(x) P_{\lambda_{j}} u(x) \\
& =\sum_{k} \frac{\phi_{k}(x)}{\lambda_{k}^{n}} \sum_{j} \widetilde{m}_{k, n}\left(\lambda_{j}\right) P_{\lambda_{j}} u(x)=\sum_{k} \frac{\phi_{k}(x)}{\lambda_{k}^{n}} \widetilde{m}_{k, n}(-\Delta) u(x),
\end{aligned}
$$

where $\widetilde{m}_{k, n}(-\Delta)$ is the pseudo-differential operator of order 0 attached to $\widetilde{m}_{k, n}$. Theorem 3.6 and the estimates (9.4) imply that $\widetilde{m}_{k, n}(-\Delta)$ is given by integration with respect to a kernel $K_{k, n}(x, y)$ that is continuous (and smooth) off the diagonal and satisfies the estimate

$$
\left|K_{k, n}(x, y)\right| \leq C \cdot R(x, y)^{-d}
$$

where $C$ is a positive constant that is independent of $k$. Then

$$
T u(x)=\int \sum_{k} \frac{\phi_{k}(x)}{\lambda_{k}^{n}} K_{k, n}(x, y) u(y) d \mu(y) .
$$

Thus it suffices to prove that

$$
K(x, y)=\sum_{k} \frac{\phi_{k}(x)}{\lambda_{k}^{n}} K_{k, n}(x, y)
$$

is well defined and continuous. The estimates (9.6) imply that

$$
|K(x, y)| \leq C \cdot R(x, y)^{-d} \sum_{k} \frac{\lambda_{k}^{\alpha}}{\lambda_{k}^{n}}=C S \cdot R(x, y)^{-d} .
$$

Thus $K(x, y)$ is continuous off the diagonal and (9.2) holds.

We conjecture that the kernel $K$ of a pseudo-differential operator $p(x,-\Delta)$ of order 0 is a Calderón-Zygmund operator in the sense of Section I.6.5 in [31]. Even though we are unable at this point to prove this claim, we can, nevertheless, show that these operators are bounded on $L^{q}(\mu)$, for all $1<q<\infty$.

Theorem 9.6. Suppose that $X$ is a compact fractafold with no boundary and $p \in S^{0}$. Then the operator $T u(x)=p(x,-\Delta) u(x)$ extends to a bounded operator on $L^{q}(\mu)$ for all $1<q<\infty$.

Proof. Fix $1<q<\infty$ and let $r$ be such that $1 / q+1 / r=1$. Let $u \in \mathcal{D}$ and let $\widetilde{m}_{k, n}$ be as in the proof of Theorem 9.5 . Recall that $\widetilde{m}_{k, n}$ is a symbol of order 0 with constant coefficients, and

$$
T u(x)=\sum_{k} \frac{\phi_{k}(x)}{\lambda_{k}^{n}} \tilde{m}_{k, n}(-\Delta) u(x) \quad \text { for all } n \geq 0 .
$$


Recall from inequality (9.5) that there is $\alpha$ such that $\left\|\phi_{k}\right\|_{\infty} \leq c \lambda_{k}^{\alpha}$ for some positive constant $c$. Let $n \geq 0$ be such that the series $S_{1}=\sum_{k} \lambda_{k}^{r(\alpha-n / 2)}$ and $S_{2}=\sum_{k} \lambda_{k}^{-n q / 2}$ converge. The estimates (9.4) and Corollary 3.7 imply that $\widetilde{m}_{k, n}(-\Delta)$ is a bounded operator on $L^{q}(\mu)$ with a bound independent of $k$. Then, the Hölder inequality implies that

$$
\begin{aligned}
\|T u\|_{q}^{q} & =\int\left|\sum_{k} \frac{\phi_{k}(x)}{\lambda_{k}^{n}} \widetilde{m}_{k, n}(-\Delta) u(x)\right|^{q} d \mu(x) \\
& \leq \int\left(\sum_{k}\left|\frac{\phi_{k}(x)}{\lambda_{k}^{n / 2}} \frac{\widetilde{m}_{k, n}(-\Delta) u(x)}{\lambda_{k}^{n / 2}}\right|\right)^{q} d \mu(x) \\
& \leq \int\left(\sum_{k} \frac{\left|\phi_{k}(x)\right|^{r}}{\lambda_{k}^{n r / 2}}\right)^{q / r} \cdot\left(\sum_{k} \frac{\left|\widetilde{m}_{k, n}(-\Delta) u(x)\right|^{q}}{\lambda_{k}^{n q / 2}}\right) d \mu(x) \\
& \leq c^{q} \int\left(\sum_{k} \lambda_{k}^{r(\alpha-n / 2)}\right)^{q / r} \sum_{k} \frac{\left|\widetilde{m}_{k, n}(-\Delta) u(x)\right|^{q}}{\lambda_{k}^{n q / 2}} d \mu(x) \\
& =c^{q} S_{1} \sum_{k} \frac{1}{\lambda_{k}^{n q / 2}} \int\left|\widetilde{m}_{k, n}(-\Delta) u(x)\right|^{q} d \mu(x) \leq C(n) S_{1} S_{2}\|u\|_{q}^{q} .
\end{aligned}
$$

Thus $T$ extends to a bounded operator on $L^{q}(\mu)$.

\section{References}

[1] Allan, A., Barany, M. and Strichartz, R. S.: Spectral operators on the Sierpiński gasket. I. Complex Var. Elliptic Equ. 54 (2009), no. 6, 521-543.

[2] Barlow, M. T. And Bass, R. F.: The construction of Brownian motion on the Sierpiński carpet. Ann. Inst. H. Poincaré Probab. Statist. 25 (1989), no. 3, 225-257.

[3] Barlow, M.T. ANd BAss, R. F.: Brownian motion and harmonic analysis on Sierpiński carpets. Canad. J. Math. 51 (1999), no. 4, 673-744.

[4] Barlow, M. T. And Perkins, E. A.: Brownian motion on the Sierpiński gasket. Probab. Theory Related Fields 79 (1988), no. 4, 543-623.

[5] Ben-Bassat, O., Strichartz, R. S. and Teplyaev, A.: What is not in the domain of the Laplacian on Sierpiński gasket type fractals. J. Funct. Anal. 166 (1999), no. 2, 197-217.

[6] Bockelman, B. And Strichartz, R. S.: Partial differential equations on products of Sierpiński gaskets. Indiana Univ. Math. J. 56 (2007), no. 3, 1361-1375.

[7] Constantin, S., Strichartz, R. S. and Wheeler, M.: Analysis of the Laplacian and spectral operators on the Vicsek set. Commun. Pure Appl. Anal. 10 (2011), no. 1, 1-44.

[8] Davies, E. B.: Heat kernels and spectral theory. Cambridge Tracts in Mathematics 92, Cambridge University Press, Cambridge, 1990.

[9] Davies, E. B.: Uniformly elliptic operators with measurable coefficients. J. Funct. Anal. 132 (1995), no. 1, 141-169.

[10] Drenning, S. and Strichartz, R. S.: Spectral decimation on Hambly's homogeneous hierarchical gaskets. Illinois J. Math. 53 (2009), no. 3, 915-937. 
[11] Duong, X. T., Ouhabaz, E. M. and Sikora, A.: Plancherel-type estimates and sharp spectral multipliers. J. Funct. Anal. 196 (2002), no. 2, 443-485.

[12] Fitzsimmons, P. J., Hambly, B. M. and Kumagai, T.: Transition density estimates for Brownian motion on affine nested fractals. Comm. Math. Phys. 165 (1994), no. $3,595-620$.

[13] Fukushima, M. And Shima, T.: On a spectral analysis for the Sierpiński gasket. Potential Anal. 1 (1992), no. 1, 1-35.

[14] Hambly, B. M. and Kumagai, T.: Transition density estimates for diffusion processes on post critically finite self-similar fractals. Proc. London Math. Soc. (3) 78 (1999), no. 2, 431-458.

[15] Hambly, B. M. and Kumagai, T.: Diffusion processes on fractal fields: heat kernel estimates and large deviations. Probab. Theory Related Fields 127 (2003), no. 3, 305-352.

[16] Hebisch, W.: Sharp pointwise estimate for the kernels of the semigroup generated by sums of even powers of vector fields on homogeneous groups. Studia Math. 95 (1989), no. 1, 93-106.

[17] Hörmander, L.: The analysis of linear partial differential operators. III. Pseudo-differential operators. Grundlehren der Mathematischen Wissenschaften 274, Springer Verlag, Berlin, 1985.

[18] Hutchinson, J. E.: Fractals and self-similarity. Indiana Univ. Math. J. 30 (1981), no. $5,713-747$.

[19] Ionescu, M. and Rogers, L.: Complex powers of the Laplacian on affine nested fractals as Calderón-Zygmund operators. To appear in Comm. Pure Appl. Anal.

[20] Ionescu, M., Pearse, E. P. J., Rogers, L. G., Ruan, H. J. And StriCHARTz, R.S.: The resolvent kernel for p.c.f. self-similar fractals. Trans. Amer. Math. Soc. 362 (2010), no. 8, 4451-4479.

[21] Kigami, J.: Analysis on fractals. Cambridge Tracts in Mathematics 143, Cambridge University Press, Cambridge, 2001.

[22] Lindstrøм, T.: Brownian motion on nested fractals. Mem. Amer. Math. Soc. 83 (1990), no. 420, iv+128.

[23] Needleman, J., Strichartz, R. S., Teplyaev, A. and Yung, P. L.: Calculus on the Sierpiński gasket. I. Polynomials, exponentials and power series. J. Funct. Anal. 215 (2004), no. 2, 290-340.

[24] Rogers, L. G.: Estimates for the resolvent kernel of the Laplacian on p.c.f. self similar fractals and blow-ups. Trans. Amer. Math. Soc. 364 (2012), no. 3, 16331685 .

[25] Rogers, L. G. And Strichartz, R. S.: Distribution theory on p.c.f. fractals. J. Anal. Math. 112 (2010), 137-191.

[26] Rogers, L. G., Strichartz, R. S. and Teplyaev, A.: Smooth bumps, a Borel theorem and partitions of smooth functions on p.c.f. fractals. Trans. Amer. Math. Soc. 361 (2009), no. 4, 1765-1790.

[27] Sabot, C.: Pure point spectrum for the Laplacian on unbounded nested fractals. J. Funct. Anal. 173 (2000), no. 2, 497-524.

[28] Sikora, A.: Multivariable spectral multipliers and analysis of quasi-elliptic operators on fractals. Indiana Univ. Math. J. 58 (2009), no. 1, 317-334. 
[29] Sogge, C. D.: Fourier integrals in classical analysis. Cambridge Tracts in Mathematics 105, Cambridge Univ Press, Cambridge, 1993.

[30] Stein, E. M.: Singular integrals and differentiability properties of functions. Princeton Mathematical Series 30, Princeton University Press, Princeton, NJ, 1970.

[31] Stein, E. M.: Harmonic analysis: real-variable methods, orthogonality, and oscillatory integrals. Princeton Mathematical Series 43, Monographs in Harmonic Analysis III, Princeton University Press, Princeton, NJ, 1993.

[32] Strichartz, R.S. And Teplyaev, A.: Spectral analysis on infinite Sierpiński fractafolds. J. Anal. Math. 116 (2012), 255-297.

[33] Strichartz, R. S.: $L^{p}$ estimates for integral transforms. Trans. Amer. Math. Soc. 136 (1969), 33-50.

[34] Strichartz, R. S.: Fractals in the large. Canad. J. Math. 50 (1998), no. 3, 638-657.

[35] Strichartz, R. S.: Fractafolds based on the Sierpiński gasket and their spectra. Trans. Amer. Math. Soc. 355 (2003), no. 10, 4019-4043 (electronic).

[36] Strichartz, R. S.: Function spaces on fractals. J. Funct. Anal. 198 (2003), no. 1, 43-83.

[37] Strichartz, R. S.: Analysis on products of fractals. Trans. Amer. Math. Soc. 357 (2005), no. 2, 571-615 (electronic).

[38] Strichartz, R.S.: Laplacians on fractals with spectral gaps have nicer Fourier series. Math. Res. Lett. 12 (2005), no. 2-3, 269-274.

[39] Strichartz, R. S.: Differential equations on fractals. A tutorial. Princeton University Press, Princeton, NJ, 2006.

[40] Strichartz, R. S.: A fractal quantum mechanical model with Coulomb potential. Commun. Pure Appl. Anal. 8 (2009), no. 2, 743-755.

[41] Taylor, M. E.: Pseudo-differential operators. Princeton Mathematical Series 34, Princeton University Press, Princeton, NJ, 1981.

[42] Teplyaev, A.: Spectral analysis on infinite Sierpiński gaskets. J. Funct. Anal. 159 (1998), no. 2, 537-567.

Received August 31, 2011; revised May 2, 2012.

Marius Ionescu: Department of Mathematics, Colgate University, NY 13346, USA.

E-mail: mionescu@colgate.edu

Luke G. Rogers: Department of Mathematics, University of Connecticut, Storrs, CT 06269, USA.

E-mail: rogers@math.uconn.edu

Robert S. Strichartz: Department of Mathematics, Cornell University, Ithaca, NY 14853, USA.

E-mail: str@math.cornell.edu

The work of the first author was partially supported by a grant from the Simons Foundation (\#209277 to Marius Ionescu). The work of the third author was partially supported by the National Science Foundation, grant DMS-0652440. 